\title{
New Insights on Planet Formation in WASP-47 from a Simultaneous Analysis of Radial Velocities and Transit Timing Variations
}

\author{
Lauren M. Weiss ${ }^{1,9}$, Katherine M. Deck ${ }^{2}$, Evan Sinukoff ${ }^{3,10}$, Erik A. Petigura ${ }^{2,11}$, Eric Agol ${ }^{4,5}$, Eve J. Lee ${ }^{6}$, Juliette C. Becker ${ }^{7}$, \\ Andrew W. Howard ${ }^{2}$, Howard Isaacson ${ }^{6}$, Ian J. M. Crossfield ${ }^{8}$, Benjamin J. Fulton ${ }^{3}$, Lea Hirsch ${ }^{6}$, and Björn Benneke ${ }^{2}$ \\ ${ }^{1}$ Institut de Recherche sur les Exoplanètes, Université de Montréal, Montréal, QC, Canada \\ ${ }^{2}$ California Institute of Technology, Pasadena, CA, USA \\ ${ }^{3}$ Institute for Astronomy, University of Hawai ‘i at Mānoa, Honolulu, HI, USA \\ ${ }^{4}$ Astronomy Department, University of Washington, Seattle, WA, USA \\ ${ }^{5}$ NASA Astrobiology Institute Virtual Planet Laboratory, Seattle, WA, USA \\ ${ }_{7}^{6}$ Astronomy Department, University of California, Berkeley, CA, USA \\ ${ }^{7}$ Astronomy Department, University of Michigan, Ann Arbor, MI, USA \\ ${ }^{8}$ Astronomy Department, University of California, Santa Cruz, CA, USA \\ Received 2016 December 14; revised 2017 March 22; accepted 2017 March 23; published 2017 May 25
}

\begin{abstract}
Measuring precise planet masses, densities, and orbital dynamics in individual planetary systems is an important pathway toward understanding planet formation. The WASP-47 system has an unusual architecture that motivates a complex formation theory. The system includes a hot Jupiter ("b") neighbored by interior ("e") and exterior ("d") sub-Neptunes, and a long-period eccentric giant planet ("c"). We simultaneously modeled transit times from the Kepler $K 2$ mission and 118 radial velocities to determine the precise masses, densities, and Keplerian orbital elements of the WASP-47 planets. Combining RVs and TTVs provides a better estimate of the mass of planet $\mathrm{d}\left(13.6 \pm 2.0 M_{\oplus}\right)$ than that obtained with only RVs $\left(12.75 \pm 2.70 M_{\oplus}\right)$ or TTVs $\left(16.1 \pm 3.8 M_{\oplus}\right)$. Planets e and d have high densities for their size, consistent with a history of photoevaporation and/or formation in a volatile-poor environment. Through our RV and TTV analysis, we find that the planetary orbits have eccentricities similar to the solar system planets. The WASP-47 system has three similarities to our own solar system: (1) the planetary orbits are nearly circular and coplanar, (2) the planets are not trapped in mean motion resonances, and (3) the planets have diverse compositions. None of the current single-process exoplanet formation theories adequately reproduce these three characteristics of the WASP-47 system (or our solar system). We propose that WASP-47, like the solar system, formed in two stages: first, the giant planets formed in a gas-rich disk and migrated to their present locations, and second, the high-density sub-Neptunes formed in situ in a gas-poor environment.
\end{abstract}

Key words: planets and satellites: composition - planets and satellites: formation - planets and satellites: general planets and satellites: individual (WASP-47 b, WASP-47 c, WASP-47 d, WASP-47 e)

Supporting material: data behind figures

\section{Introduction}

One of the key questions driving exoplanet science is the formation of planetary systems in general and the solar system in particular. The Kepler mission (Borucki et al. 2010; Koch et al. 2010) led to a wealth of statistical measurements that provide valuable insight into planet formation. Small planets close to their stars are a common outcome of planet formation (Fang \& Margot 2012; Howard et al. 2012; Batalha et al. 2013; Fressin et al. 2013; Petigura et al. 2013b; Dressing \& Charbonneau 2015), such that half of Sun-like stars have at least one planet smaller than Neptune within the orbital distance of Mercury (Petigura et al. 2013a; Burke et al. 2015). Many small planets close to their stars are in multi-planet systems (Lissauer et al. 2012, 2014; Fabrycky et al. 2014; Rowe et al. 2014). Given the abundance of small, compact planetary systems around other stars, is our own solar system unusual in that it is barren from Mercury's orbit inward? Because the Kepler mission only obtained 4 years of continuous photometry and only observed 150,000 stars, it

\footnotetext{
9 Trottier Fellow.

${ }^{10}$ NSERC Fellow.

11 NASA Hubble Fellow.
}

had poor sensitivity to long-period planets, which are unlikely to transit. If Kepler were pointed at our solar system and were lucky enough to discover the inner planets, it still most likely would have missed the planets from Mars outward. How can we reconcile planet formation theory for the close-in exoplanets with planet formation theory for our spaciously spread solar system?

WASP-47 is a system with an unusual architecture that might be a Rosetta Stone for linking the exoplanet population to the solar system. WASP-47 contains a transiting, Jupiter-size planet with an orbital period of 4.2 days (a "hot Jupiter") that was detected from the ground-based WASP-South transit survey (Hellier et al. 2012, WASP-47 b). What makes WASP-47 b unusual is that, contrary to the vast majority of hot Jupiters, which do not have nearby planetary companions (Steffen et al. 2012; Bryan et al. 2016), WASP-47 b has two nearby neighbors: an interior, transiting planet with an orbital period of less than a day (WASP-47 e) and an exterior, transiting planet with an orbital period of 9.0 days (WASP-47 d). The system also has a distant, moderately eccentric planet (WASP-47 c). While the compactness of the WASP-47 inner planetary system is comparable to other Kepler systems, especially those that contain ultra-short period planets 
(Sanchis-Ojeda et al. 2013), the combination of the compact planetary system with a hot Jupiter is unprecedented among the $2217^{12}$ planetary systems studied to date. The architecture of WASP-47 was not predicted by planet formation theory, so uncovering a physically plausible formation mechanism for WASP-47 will deepen our understanding of planet formation in general.

To better understand which of the various physical models of planet formation and evolution were important in the WASP-47 system, we would like to measure the masses, densities, bulk compositions, and orbital dynamics of all the planets as precisely as the current data permit. The compositions of the planets might provide clues about where they formed within the protoplanetary disk - for instance, if the planets are rich in water or other high mean-molecular weight volatiles, they might have formed beyond a molecular snowline. Furthermore, the present-day orbital elements for the planets can be related to their dynamical history: the semimajor axes and eccentricities of the planets today relate to how they have exchanged energy and angular momentum in the past.

Several analyses of this system have already characterized various dynamical properties of the WASP-47 planets. The discovery paper (Hellier et al. 2012) used two years of groundbased photometry to find WASP-47 b in transit, and also obtained 19 low-precision radial velocities (RVs) to measure the planet's mass. Becker et al. (2015, hereafter B15) discovered two additional transiting planets (e and d) in transits from $\mathrm{K} 2$, characterized the planet masses with transit timing variations (TTVs), and used the Mercury $N$-body integrator (Chambers 1999) to explore the dynamical stability of the planets. Sanchis-Ojeda et al. (2015) measured the projected spin-orbit obliquity of the hot Jupiter via the RossiterMcLaughlin effect, finding that the planetary orbital axis and the stellar spin axis are not strongly misaligned. Dai et al. (2015) obtained high-cadence precision RVs of the system, precisely characterizing the mass of the giant planet and placing new mass constraints on the other transiting planets. Neveu-VanMalle et al. (2016) discovered a long-period giant planet with a multi-year baseline of radial velocities. Almenara et al. (2016) simultaneously modeled the $K 2$ light curve and the literature RVs, arriving at planet masses that were determined to a precision of $\sim 40 \%$. Sinukoff et al. (2017, hereafter S17) obtained 47 new RVs with Keck-HIRES, which, when combined with the literature RVs, significantly improved the precision of the mass and $M_{\mathrm{p}} \sin i$ measurements $(<25 \%)$ for all the WASP-47 planets.

We present a robust analysis of the planet masses and orbital dynamics by combining the 108 transit times measured in B15 with 118 literature radial velocities. Our paper is structured as follow. In Section 2 we introduce the measurements analyzed herein. In Section 3 we present two ways to analyze the TTVs alone: using an $\mathrm{N}$-body integrator and a dynamical analytic solver. In Section 4 we present a joint analysis of the TTVs and RVs of the WASP-47 system that results in the most accurate and precise dynamical parameters to date. In Section 5 we present a new, simple way to combine information from TTVs and RVs. In Section 6 we discuss how our improved mass and eccentricity information relates to planet formation theory. We conclude in Section 7.

\footnotetext{
${ }^{12}$ Based on a 2016 November 24 query of exoplanets.org.
}

\section{Measurements}

The measurements we use in this analysis are all available in the literature. We combine the 108 transit times (TTs or TTVs) of WASP-47 e, b, and d (B15) with 118 measurements of the radial velocity (RV) of the WASP-47 host star from Hellier et al. (2012), Dai et al. (2015), Neveu-VanMalle et al. (2016), and S17.

To combine the RV measurements, we use the values for the RV zero-point offset $(\gamma)$ and jitter $\left(\sigma_{\text {jit }}\right)$ determined in S17. The zero-point offset is added to each RV measurement, and the jitter is added to each RV uncertainty in quadrature. For the Hellier et al. (2012) CORALIE RVs, these values are $\gamma=27070.3 \mathrm{~m} \mathrm{~s}^{-1}, \sigma_{\mathrm{jit}}=5.9 \mathrm{~m} \mathrm{~s}^{-1}$. For the Neveu-VanMalle et al. (2016) CORALIE RVs, these values are $\gamma=$ $27085.3 \mathrm{~m} \mathrm{~s}^{-1}, \sigma_{\mathrm{jit}}=6.7 \mathrm{~m} \mathrm{~s}^{-1}$. For the Dai et al. (2015) Magellan-PFS RVs, these values are $\gamma=20.5 \mathrm{~m} \mathrm{~s}^{-1}$, $\sigma_{\text {jit }}=6.3 \mathrm{~m} \mathrm{~s}^{-1}$. For the S17 Keck-HIRES RVs, these values are $\gamma=6.4 \mathrm{~m} \mathrm{~s}^{-1}, \sigma_{\mathrm{jit}}=3.7 \mathrm{~m} \mathrm{~s}^{-1}$. For simplicity, we keep the values of the zero-point offset and jitters fixed at the values determined in S17. The zero-point offsets and jitter are statistical properties of the RVs, so we do not expect the TTVs to provide any new information about these parameters.

\section{TTVs-only Analysis with N-body and Analytic Approaches}

In this section, we fit the WASP-47 TTVs as measured in B15 using two different approaches. First, we do a full $N$-body simulation of the three transiting planets to reproduce the observed TTVs using the publicly available code TTVFast (Deck et al. 2014). Then, we use TTVFaster (Agol \& Deck 2016a, 2016b), a publicly available code that analytically models orbits to first order in eccentricity. The TTVFaster code was designed to reproduce both low-frequency sinusoidal features and high-frequency "chopping" patterns in the TTVs. In the tests below, we determine that TTVFaster, which is orders of magnitude faster than TTVFast, is appropriate for modeling the TTVs in the WASP-47 system.

\subsection{Modeling Transit Times with an N-body Integrator}

We used the publicly available $N$-body integrator TTVFast (Deck et al. 2014) with the python wrapper ttvfastpython ${ }^{13}$ to forward-model the transit times of the inner three planets. Unlike in the B15 analysis, we allowed all of the initial osculating elements, particularly the orbital periods and initial times of transits, to vary. Thus, the variables for each planet $k$ are: the mass of the planet $M_{k}$, the orbital period $P_{k}$, the first time of transit $t t_{k}$, and the eccentricity parametrization $\sqrt{e_{k}}$ $\cos \omega_{k}, \sqrt{e_{k}} \sin \omega_{k}$. We limited $e<0.06$ for the three inner planets, in accordance with the $10 \mathrm{Myr}$ stability analysis in B15. For each adjacent pair of planets, we satisfied the Hill criteria for stability for low-eccentricity orbits, as described in Equations (24) and (28) of Gladman (1993):

$$
\begin{gathered}
p-q>2.4\left(\mu_{1}^{2}+\mu_{2}^{2}\right)^{1 / 3}, \\
p-q>\sqrt{8 / 3\left(e_{1}^{2}+e_{2}^{2}\right)+9 \times \max \left(\mu_{1}, \mu_{2}\right)^{2 / 3}},
\end{gathered}
$$

where the subscript 1 refers to the inner planet and 2 refers to the outer planet, $q$ is the apoapse distance of the inner planet

\footnotetext{
${ }^{13}$ https://github.com/mindriot101/ttvfast-python
} 
Table 1

Priors on Dynamical Parameters

\begin{tabular}{ll}
\hline \hline Parameter & Priors \\
\hline$M$ & $M>0$, Hill criterion \\
$P$ & $P>0$, Hill criterion \\
$T T$ & None \\
$\sqrt{e} \cos \omega$ & $e<0.06$, Hill criterion \\
$\sqrt{e} \sin \omega$ & $e<0.06$, Hill criterion \\
$\sqrt{e_{c}} \cos \omega_{c}$ & $e<1$, Hill criterion \\
$\sqrt{e_{c}} \sin \omega_{c}$ & $e<1$, Hill criterion \\
\hline
\end{tabular}

$q=1+e_{1}, p$ is the periapse distance of the outer planet $p=\left(1-e_{2}\right) \frac{a_{2}}{a_{1}}, e$ is the eccentricity, and $\mu$ is the planet-tostar mass ratio. We also required the planet masses and orbital periods to have positive values. Because the orbits of the planets are very nearly coplanar (Becker et al. 2015; Almenara et al. 2016), we fixed the orbital inclination and longitude of the ascending node in a manner consistent with coplanar, edge-on orbits. See Table 1 for a summary of the priors and constraints.

We used the Markov Chain Monte Carlo (MCMC) Python package emcee (Foreman-Mackey et al. 2013) to explore the posteriors of various combinations of the dynamical parameters. We ran 60 walkers $5 \times 10^{5}$ steps each, throwing away the first $10^{5}$ steps as burn-in, and checked that the multivariate extension of the potential scale reduction factor (PSRF) statistic $(\hat{R}<1.2$, Gelman \& Rubin 1992; Brooks \& Gelman 1998) converged. We also inspected the chains by eye to check for convergence. Our best fit $^{14}$ to the transit times using TTVFast is shown in Figure 1. Table 2 summarizes our results from this $N$-body fit to the TTVs.

We find that for planets e and $b$, the masses and eccentricities of the planets are highly covariant with the initial osculating orbital period and the initial transit time of neighboring planets (see Figure 2). This is because the initial osculating orbital period is translated to an instantaneous velocity and acceleration, and the planet's acceleration depends on the mass and position of its neighbor. Therefore, it is critical to allow the initial orbital periods and times of transit of all the planets to vary in order to explore the full range of possible planet masses. Furthermore, only one super-period of the TTVs is observed, so an average orbital period and a representative time of transit are not as well determined for planets $b$ and $d$ as they might be with the observation of multiple super-periods. Thus, we find that the TTVs do not constrain the masses of WASP-47 e or WASP-47 b as tightly as what is reported in B15. Whereas B15 find $M_{b}=341_{-55}^{+73} M_{\oplus}$, we find $M_{b}=549 \pm 252 M_{\oplus}$, using the exact same TTV measurements. The mass for planet $\mathrm{e}$ reported in B15 stems from their choice of prior: they find $M_{e}<22 M_{\oplus}$, whereas we find $M_{e}=176 \pm 118 M_{\oplus}$. However, the TTVs of planet $\mathrm{b}$ place strong constraints on the mass of planet d. B15 find $M_{d}=15.2 \pm 7 M_{\oplus}$, and we find $M_{d}=16.1 \pm 3.8 M_{\oplus}$. While an analytic covariance between planet masses and the free eccentricity exists (Lithwick et al. 2012), our stability constraint that $e<0.06$ minimizes the effects of this degeneracy.

\footnotetext{
${ }^{14}$ From the MCMC maximum likelihood.
}

Table 2

Dynamical Parameters from the Best N-body Fit to TTVs Only (TTVFast)

\begin{tabular}{lcc}
\hline \hline Parameter & Median \pm Std. Dev. & Units \\
\hline$M_{e}$ & $176 \pm 118$ & $M_{\oplus}$ \\
$M_{b}$ & $549 \pm 252$ & $M_{\oplus}$ \\
$M_{d}$ & $16.1 \pm 3.8$ & $M_{\oplus}$ \\
$P_{e}$ & $0.78964 \pm 0.00002$ & days \\
$P_{b}$ & $4.150 \pm 0.006$ & days \\
$P_{d}$ & $9.12 \pm 0.05$ & days \\
$T T_{e}$ & $2146.7639 \pm 0.0008$ & BJD-2454833 \\
$T T_{b}$ & $2149.969 \pm 0.006$ & BJD-2454833 \\
$T T_{d}$ & $2155.40 \pm 0.05$ & BJD-2454833 \\
$\sqrt{e_{e}} \cos \omega_{e}$ & $-0.006 \pm 0.14$ & $\ldots$ \\
$\sqrt{e_{e}} \sin \omega_{e}$ & $-0.008 \pm 0.14$ & $\ldots$ \\
$\sqrt{e_{b}} \cos \omega_{b}$ & $0.001 \pm 0.08$ & $\ldots$ \\
$\sqrt{e_{b}} \sin \omega_{b}$ & $0.006 \pm 0.07$ & $\ldots$ \\
$\sqrt{e_{d}} \cos \omega_{d}$ & $-0.09 \pm 0.11$ & $\ldots$ \\
$\sqrt{e_{d}} \sin \omega_{d}$ & $0.07 \pm 0.09$ & $\ldots$ \\
\hline
\end{tabular}

Note. These are the initial astrocentric Keplerian orbital elements, reported at epoch BJD 2456979.4961. They are not the time-averaged orbital properties of the planets.

\subsection{Modeling Transit Times Analytically}

We used the publicly available analytic TTV package TTVFaster to model the transit times of the inner three planets observed in B15. The TTVFaster code analytically transforms the planet mass $M_{k}$ and average orbital elements $P_{k}, t t_{k}, e_{k} \cos \omega_{k}$, $e_{k} \sin \omega_{k}^{15}$ into a TTV pattern, to first order in eccentricity, to a user-specified precision in the disturbing function. We found that modeling to sixth order in the expansion of the Laplace coefficient $b_{1 / 2}^{j}(j=\{0,1,2,3,4,5,6\})$, (Murray \& Dermott 2000) was sufficient to reproduce the observed TTV signature with the same fidelity as that produced in the $\mathrm{N}$-body analysis (see Figure 1). In general, TTVFaster is designed to work for planets that (1) are not extremely close to a mean motion resonance, (2) have low eccentricities, or (3) have low masses. Because WASP-47 b is a Jupiter-mass planet, we wanted to see if TTVFaster modeled the orbital dynamics correctly.

Incorporating the priors from Table 1, we used Python packages Imfit (Newville et al. 2014) and emcee to explore the posteriors of the dynamical parameters. We ran 100 walkers 20,000 steps, throwing away the first 4000 steps as burn-in. We note that the chains converged much faster (according to the PSRF statistic) when we used TTVFaster than when we used TTVFast, because the TTVs provide better constraints on the average orbital parameters than they do on the initial orbital parameters.

The mass and eccentricity distributions we determined from the analytic solution to the observed TTVs are consistent with the $\mathrm{N}$-body model (Figure 3). Since fitting an analytic model to the TTVs is orders of magnitude faster than a full $N$-body analysis (especially when the long time baseline for RVs is required), we use the analytic modeling technique for the rest of this paper.

\footnotetext{
$\overline{15}$ We used jump parameters $\sqrt{e_{k}} \cos \omega_{k}, \sqrt{e_{k}} \sin \omega_{k}$ to avoid an eccentricity bias and speed convergence.
} 

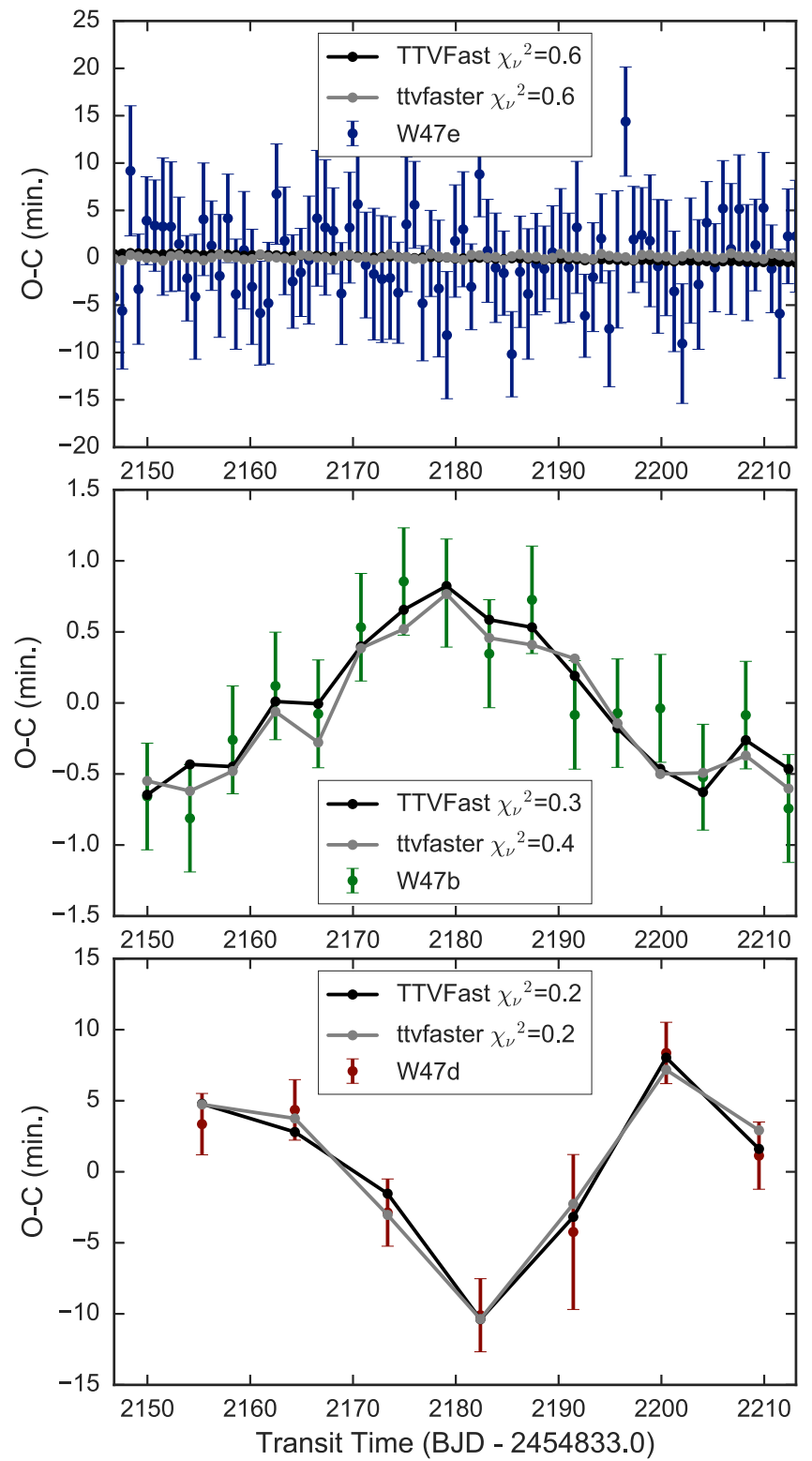

Figure 1. Observed minus linear-ephemeris calculated transit times of WASP47 e (blue points, top panel), b (green points, middle panel), and d (brown points, bottom panel). The best-fit $N$-body model to the TTVs alone (using TTVFast, black connected dots) and the best-fit analytic model to the TTVs alone (using TTVFaster, gray connected dots) are shown.

\section{Combining TTVs and RVs with TTVFaster}

We combined the python packages TTVFaster and radvel (B. J. Fulton \& E. A. Petigura 2017, in preparation ${ }^{16}$ ) to simultaneously fit the RVs and TTVs, resulting in refined masses and orbital properties for all four known planets. To fit the RV and TTV data simultaneously, we maximized the following log-likelihood function while satisfying our priors:

$$
\begin{aligned}
\ln L= & -\sum_{i}^{N_{\mathrm{RV}}} \frac{\left(\mathrm{RV}_{\mathrm{obs}, i}-\mathrm{RV}_{\mathrm{Kep}, i}\right)^{2}}{2 \sigma_{\mathrm{RV}, i}^{2}} \\
& -\sum_{k}^{N_{\mathrm{pl}}} \sum_{j}^{N_{\mathrm{TT}, \mathrm{k}}} \frac{\left(\mathrm{TT}_{\mathrm{obs}, k, j}-\mathrm{TT}_{\mathrm{model}, k, j}\right)^{2}}{2 \sigma_{\mathrm{TT}, \mathrm{k}, \mathrm{j}}^{2}},
\end{aligned}
$$

\footnotetext{
${ }^{16}$ https://github.com/California-Planet-Search/radvel
}

where $N_{\mathrm{RV}}$ is the number of RVs, $N_{\mathrm{pl}}$ is the number of planets, $N_{\mathrm{TT}, k}$ is the number of transit times for planet $k, \mathrm{RV}_{\mathrm{obs}, i}$ is the $i$ th observed RV, including the instrument-specific fixed zeropoint offset $\gamma$ determined in $\mathrm{S} 17, \mathrm{RV}_{\mathrm{Kep}, i}$ is the $i$ th Keplerianmodeled $\mathrm{RV}, \sigma_{\mathrm{RV}, i}$ is the uncertainty in $\mathrm{RV}_{\mathrm{obs}, i}$, including a constant jitter for each spectrometer determined in $\mathrm{S} 17, \mathrm{TT}_{\mathrm{obs}, k, j}$ is the $j$ th observed transit time for planet $k, \mathrm{TT}_{\text {model }, k, j}$ is the $j$ th modeled transit time for planet $k$, and $\sigma_{\mathrm{TT}, k, j}$ is the uncertainty in $\mathrm{TT}_{\mathrm{obs}, k, j}$.

Our model included all four known planets. The variable parameters for each planet $k$ are: the mass of the planet $M_{k}$, the orbital period of the planet $P_{k}$, a representative time of transit $t t_{k}$, and the eccentricity parametrization $\sqrt{e_{k}} \cos \omega_{k}, \sqrt{e_{k}} \sin \omega_{k}$. Note that the argument of periapse passage, $\omega_{k}$, is for the planet, not the star. This formulation is consistent with both the TTVfaster definition and the definition in Murray \& Correia (2010) and Lovis \& Fischer (2010).

These parameters are transformed into the appropriate basis to drive a Keplerian RV model (for comparison to the RVs) and the basis used for TTVFaster computations. Note that this scheme is not possible for an $N$-body integrator, since the initial orbital elements are not the same as the time-averaged orbital elements used in a Keplerian prescription. We also required Hill stability for all the planets, as described in Equations (1) and (2). In addition, we allowed the stellar mass to vary, using the prior $M_{\star}=0.99 \pm 0.05 M_{\odot}$ from S17, in case the combined RV and TTV data added new information about the stellar mass. ${ }^{17}$ The best simultaneous fit to the TTVs and RVs is shown in Figures 4 (TTVs) and 5 (RVs).

Incorporating the priors in Table 1, we used emcee to explore the posteriors and covariances of the dynamical parameters. We ran 100 walkers 10,000 steps each, throwing away the first 4000 steps as burn-in, and found that our chains had converged based on the PSRF statistic. (The inclusion of RV data helped the chains converge faster.) The result of our MCMC analysis is shown in Figure 6. Our MCMC results are summarized in Table 3.

To compute planet densities, we utilized the precise stellar density determined by the photodynamical analysis in Almenara et al. (2016). Because the transit of the giant planet has very high signal-to-noise, the transit ingress and egress are well-resolved. The clear ingress and egress and the nearly circular orbit of the giant planet enable a precise characterization of the stellar-limb-darkening parameters and the planet impact parameter. Knowledge of these physical quantities allows a precise determination of the stellar density.

We translated the precise stellar density into precise planet densities and radii in the following manner. For each MCMC trial, we drew a random stellar density from a normal distribution $\mathcal{N}(0.999,0.015)$. We combined the stellar mass and stellar density of each trial to compute the stellar radius. For each trial, we also drew a random planet-to-star radius ratio for each planet, using the radius ratios determined in Almenara et al. (2016 see Table 3 ). We computed the planet density and radius with the following equations:

$$
R_{\mathrm{p}}=\frac{R_{\mathrm{p}}}{R_{\star}} R_{\star},
$$

\footnotetext{
17 The TTVs constrain $M_{k} / M_{\star}$, whereas the RVs constrain $M_{k} / M_{\star}^{2 / 3}$.
} 


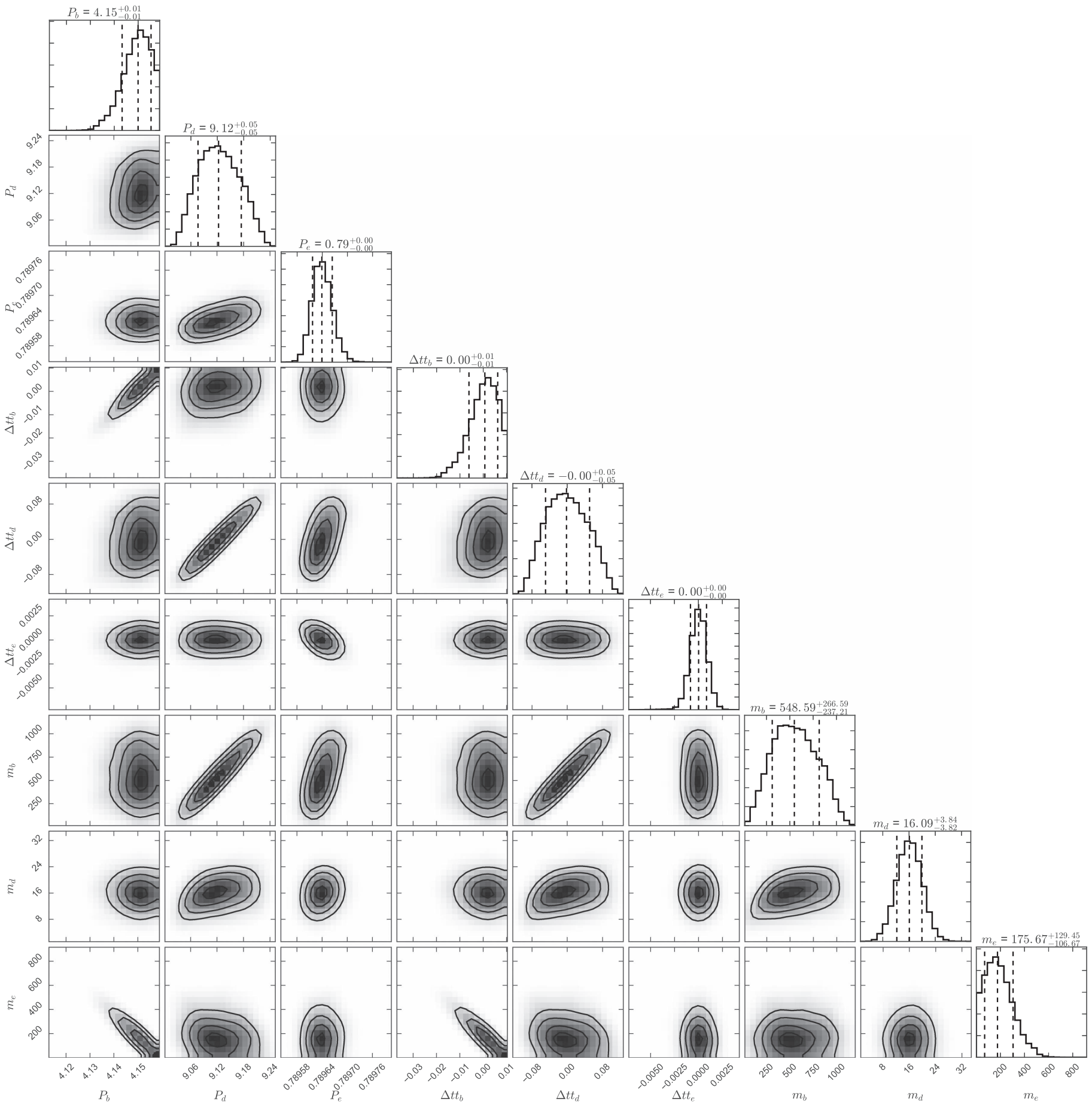

Figure 2. Posteriors of the planet masses, initial osculating orbital periods, and initial times of transit for WASP-47 e, b, and d in the TTVFast ( $N$-body) analysis. The planet masses are highly covariant with the initial orbital elements. Note that the orbital periods and times of transit here are initial osculating elements, not the timeaveraged orbital elements. The MCMC chains shown are available as data behind the figure. The data used to create this figure are available.

$$
\rho_{\mathrm{p}}=\rho_{\star}\left(\frac{M_{\mathrm{p}}}{M_{\star}}\right)\left(\frac{R_{\mathrm{p}}}{R_{\star}}\right)^{-3} .
$$

The small uncertainty in the stellar density constrains the planet densities, since the stellar mass and radius (and hence planet mass and radius) are correlated. Including the stellar density information reduces the uncertainties in the planet densities by $\sim 20 \%$. Note that this refinement of the planet densities and radii does not affect the planet masses; the masses are determined directly from TTVs and RVs. Rather, a detailed study of the stellar properties influences our interpretation of the planetary properties.

\section{Independent Multiplied Posteriors (IMPs): A Quick and Daring Way to Combine Data Sets}

In this section we offer a sanity check of our combined RV +TTV dynamical solution. Since the RVs and TTVs are 


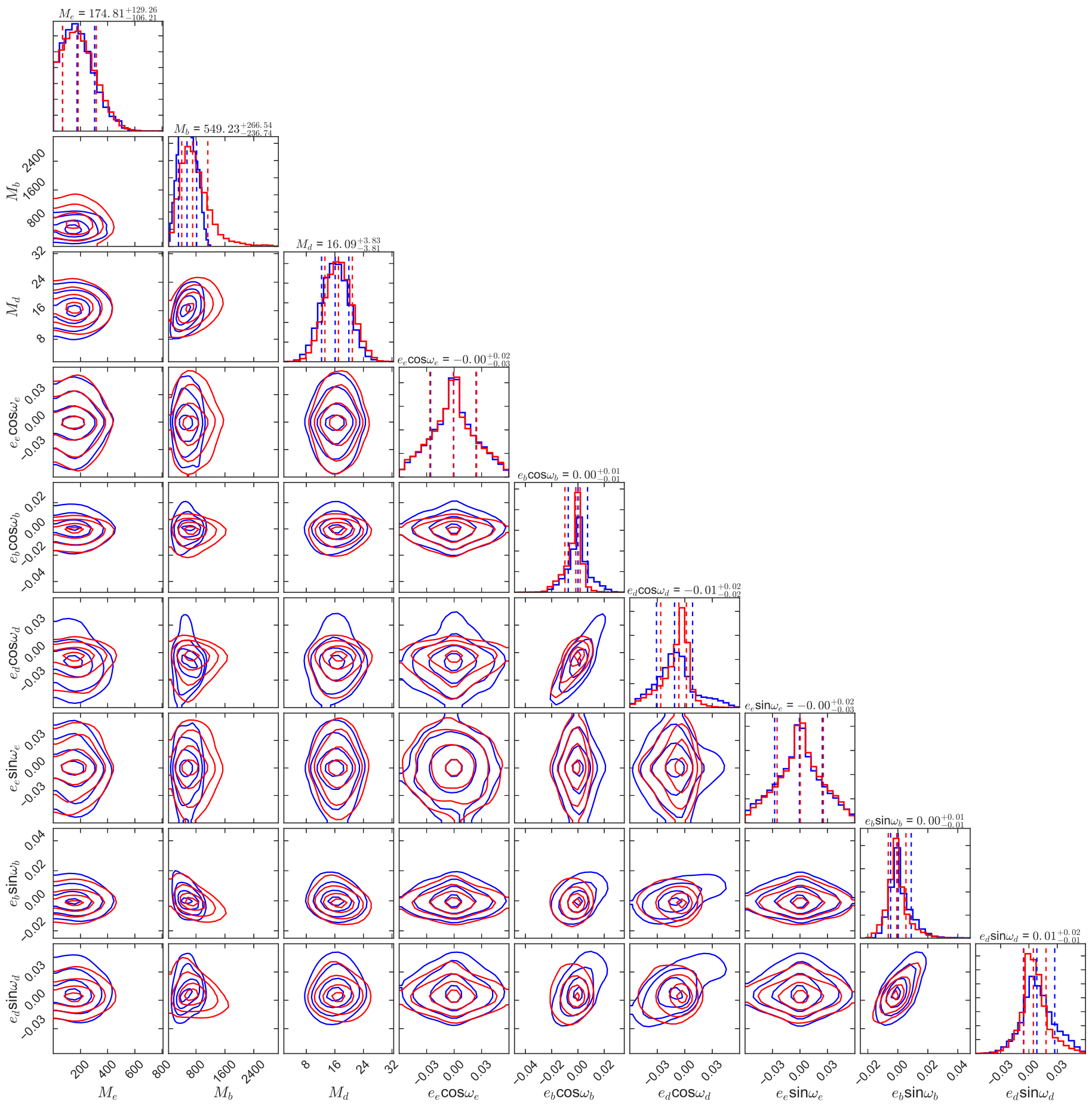

Figure 3. Posteriors of the analyses of the TTVs alone. Blue: from the TTVFast $N$-body integrator MCMC; red: from the analytic TTV modeler TTVFaster MCMC. In both analyses, we limited $e<0.06$ for the three inner planets, in accordance with the stability analysis from B15. We also required Hill stability. We used jump parameters of the form $\sqrt{e} \cos \omega$ to avoid high eccentricity biases. The mass and eccentricity posteriors obtained from the analytic TTV analysis are in good agreement with those obtained with $N$-body modeling. The values above the histograms correspond to the $N$-body posterior median and $1 \sigma$ bounds. The MCMC chains shown are available as data behind the figure. The data used to create this figure are available.

independent observations, the marginalized posteriors from their separate analyses can be multiplied together to estimate the joint probability distribution of a parameter of interest. This is codified in probability theory as

$$
P(A \cap B)=P(A) \times P(B),
$$

if $\mathrm{A}$ and $\mathrm{B}$ are independent events. Assuming that the RV time series is independent of the TTV times series, ${ }^{18}$ the posteriors of the RV-only analysis and the TTV-only analysis can be multiplied together to estimate their joint posterior. We call the

\footnotetext{
$\overline{18}$ This is easier to satisfy than the claim that each RV and each TTV are independent measurements.
} 

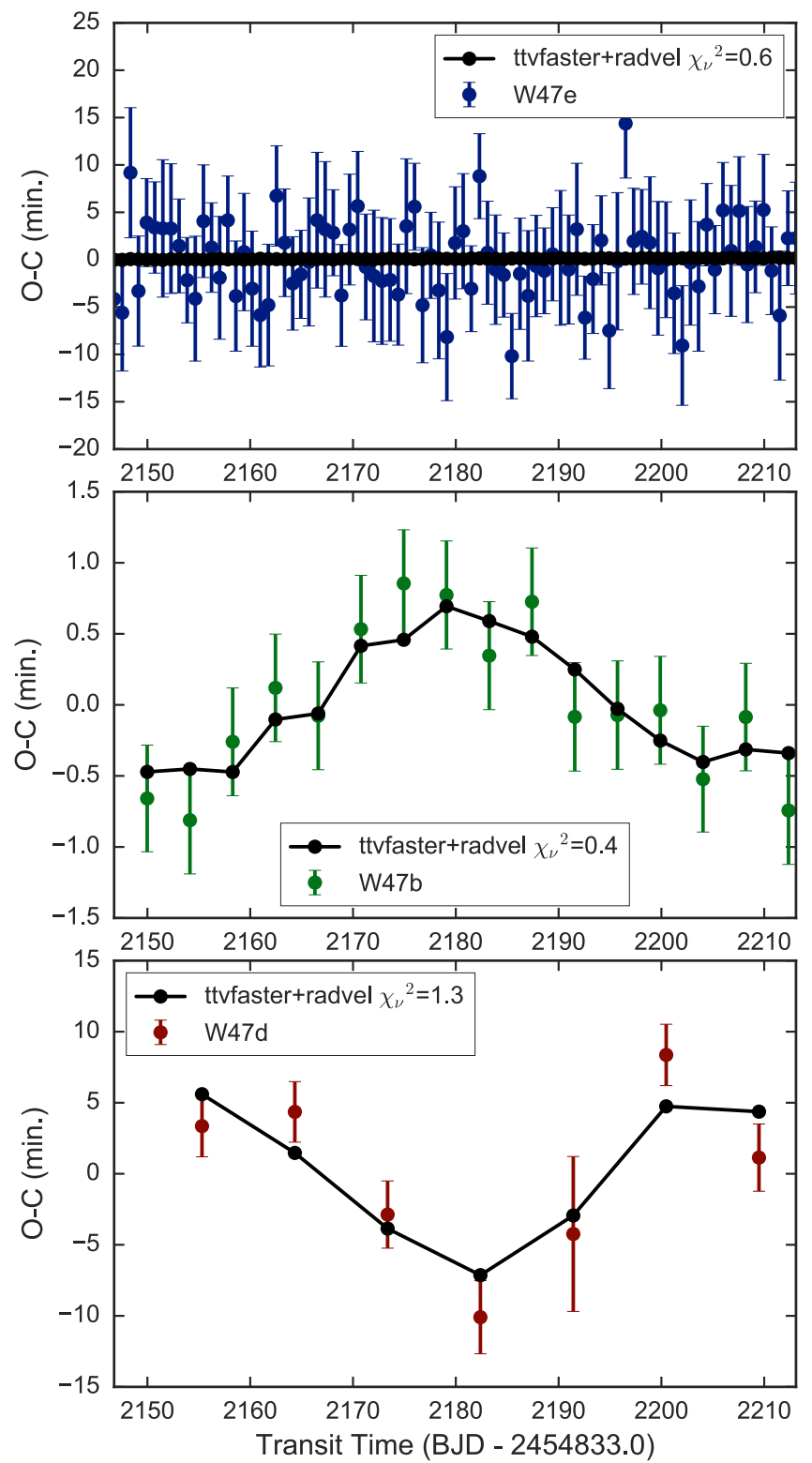

Figure 4. The best simultaneous fit to the TTVs and RVs of the WASP-47 system, and residuals. From top to bottom, the panels show the TTVs of the transiting planets e $(P=0.79$ days, blue $), \mathrm{b}(P=4.16$ days, green $)$, and $\mathrm{d}$ $(P=9.0$ days, brown). The best simultaneous fit to the TTVs + RVs of all four planets is shown as black connected points.

product of multiplying the posteriors from independent data sets an Independent Multiplied Posterior (IMP). The IMP loses some of the information of a simultaneous TTV $+\mathrm{RV}$ analysis because the RVs and TTVs are interleaved in time, and because any subtle, slight covariances in the posteriors are not captured in the IMP.

In Figure 7, we show the posteriors of the planet masses from the RV-only (S17) and TTV-only (using the $N$-body integrator) analyses, and the result of multiplying these posteriors together. For planets e and $b$, the TTVs provide no new information, so the IMPs reflect the mass posteriors from the RV-only analysis. However, the TTVs alone do measure the mass of planet d. For planet d, the IMP performs as we would expect: it peaks at a value between the RV-only and TTV-only analyses, and its width is narrower than either analysis is alone. The IMP for the mass of planet $d$ gives
$M_{d}=13.8 \pm 2.2 M_{\oplus}$. This is in good agreement with what we determined in the simultaneous modeling of the RVs+TTVs $\left(M_{d}=13.6 \pm 2.0 M_{\oplus}\right)$. As expected, the constraint we get from simultaneous modeling of the RVs+TTVs is slightly tighter than the constraint from the IMP. Also, the result of simultaneous modeling is slightly closer to the RVs-only solution $\left(M_{d}=12.75 \pm 2.70 M_{\oplus}\right)$ than the TTVs-only solution $\left(M_{d}=16.1 \pm 3.8 M_{\oplus}\right)$.

In cases where one is computation-limited or short on time, the IMP provides an approximate answer. However, if the posteriors are highly covariant in both data sets, the IMP might grossly overestimate the uncertainties and might also lose accuracy. This method for combining data sets is convenient and potentially useful for combining TTV and/or RV data sets with data from GAIA or WFIRST in the future, but should be used with great caution.

\section{Discussion}

Here we examine how our joint analysis of the WASP-47 TTVs and RVs provides information about the compositions, orbital dynamics, and formation history of the WASP-47 planets.

\subsection{Relative Information in Dynamical Analyses}

Table 4 summarizes the relative information in various dynamical analyses of the planet masses and eccentricities. B15 modeled the $K 2$ TTVs in a three-planet $N$-body analysis in which the orbital periods and initial times of transit were fixed, resulting in narrow posteriors for the mass of planet $b$. The mass constraint for planet e comes from the choice of prior, rather than the TTVs. B15 also forward-modeled the system for 10 Myr using Mercury (Chambers 1999) to ensure stability, which resulted in the tight eccentricity constraints for the inner planets: $e_{k}<0.06$.

Almenara et al. (2016, A16 hereafter) performed a photodynamical analysis of the K2 TTVs and 71 literature RVs from the PFS and CORALIE spectrographs. The high signal-to-noise of the transits of planet $b$ allowed them to determine stellarlimb-darkening parameters and the planet impact parameter very precisely, which, in combination with the small eccentricity of planet $b$, led to a very precise determination of the stellar density through asterodensity profiling (Kipping 2014). This led to a model-independent estimate of the planetary masses, presented in column A16.1 of Table 4. By including constraints from the Dartmouth stellar isochrone models (Dotter et al. 2008), the authors were able to constrain the star and planet masses more precisely, but at the expense of accuracy. The model-dependent star and planet masses are shown in column A16.2.

S17 combined 47 new HIRES RVs with the 71 literature RVs. While the CORALIE RVs had provided a long enough baseline to detect the non-transiting planet c (Neveu-VanMalle et al. 2016) and the PFS RVs had provided sufficiently high precision to detect a marginal RV signal from planet e (Dai et al. 2015), the HIRES RVs provided both a long baseline and high precision in a single data set. The HIRES data combined with the other RV data sets resulted in smaller uncertainties for all the planet masses than what had been reported in previous RV studies.

Our TTV-only $N$-body analysis (TTVs-Nbody) and simultaneous RV and TTV analysis (RVs+TTVs) are shown in 

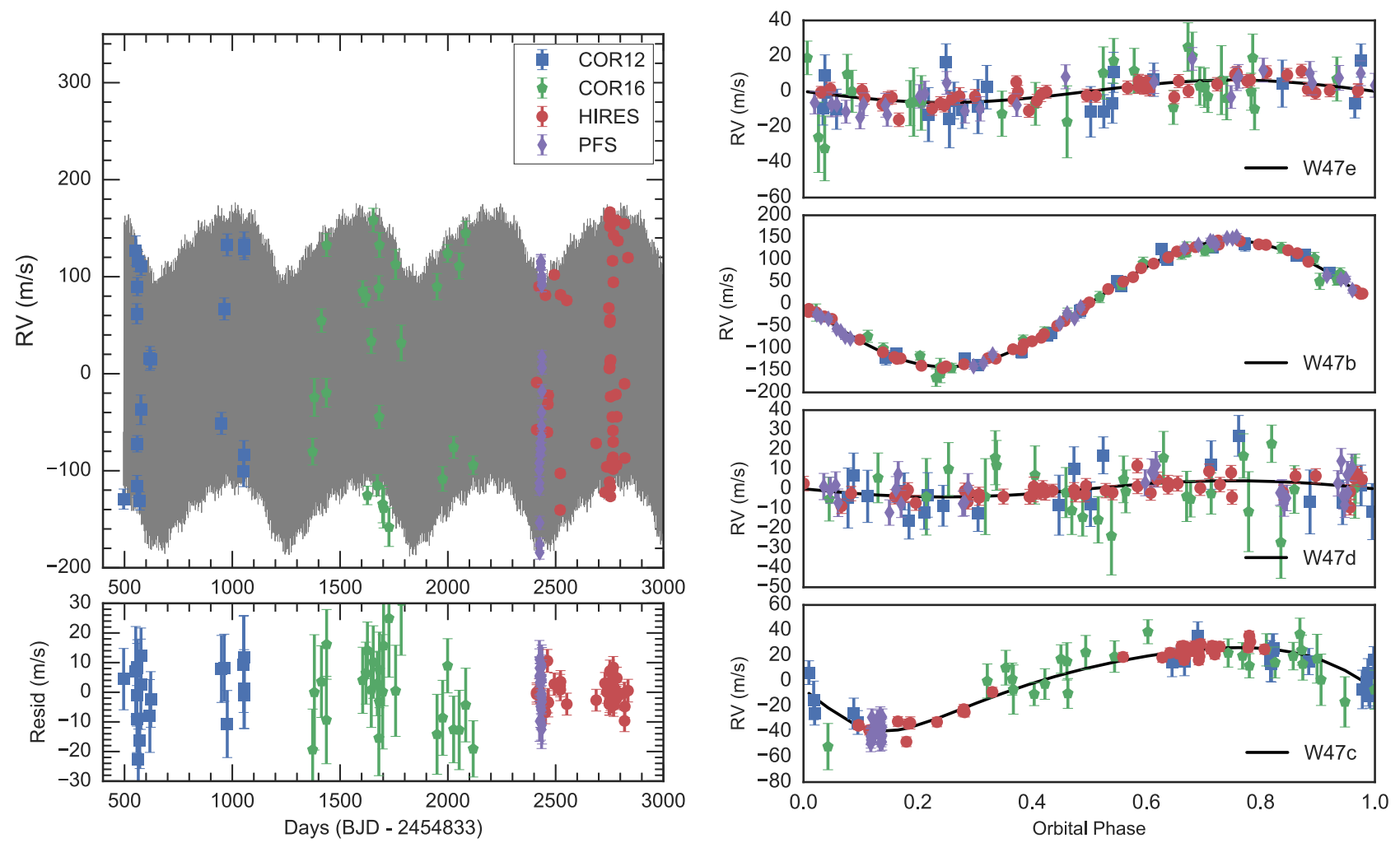

Figure 5. Left, top: radial velocities of WASP-47 from four observational campaigns: CORALIE before 2012 (blue squares), CORALIE before 2016 (green pentagons), PSF (purple diamonds), and HIRES (red circles). The best-fit model to the TTVs and RVs (fine gray line) is shown. Left, bottom: RV residuals (observations minus the TTVFaster+radvel model values). The rms of the residuals is $8.5 \mathrm{~m} \mathrm{~s}^{-1}$, which is comparable to the mean jitter-enhanced RV uncertainty over all the telescopes $\left(7.7 \mathrm{~m} \mathrm{~s}^{-1}\right.$ ). Right: the RVs phase-folded to the orbital periods of planet e (top), b (second from top), $\mathrm{d}$ (second from bottom), and c (bottom). The HIRES RVs are the only single data set that constrain the semi-amplitudes of all the planets, because they have the precision $\left(3 \mathrm{~m} \mathrm{~s}^{-1}\right)$ to capture the small amplitudes of planets e and d, and also the baseline to capture the amplitude of the long-period planet c.

Table 4. The TTVs-Nbody column illustrates how much information is contained in the TTVs. The RVs+TTVs column illustrates how much information is gained from a joint analysis of the TTVs and RVs. Our RVs+TTVs analysis confirms the precise values obtained by A16 when they include constraints from stellar models (A16.2).

How much information about planet masses comes from the TTVs? As discussed in the TTV-only analysis (see Section 3), the TTVs do not provide much information about the transiting planet masses, with the exception of the mass of planet $\mathrm{d}$, which is constrained through the TTVs of planet $b$.

Simultaneously modeling the TTVs and RVs of planet d yields a more precise determination of the mass of planet $d$ than can be obtained from either analysis alone: the uncertainties shrink from 3.8 $M_{\oplus}$ (TTVs) and $2.7 M_{\oplus}$ (RVs) to $2.0 M_{\oplus}$ (TTVs $+\mathrm{RVs})$. The TTVs provide no information about the mass of planet $\mathrm{c}$, which has a very long period compared to the inner planetary system and thus has no effect on the TTVs. Thus, the RVs provide the majority of the information about planet masses, although the TTVs contribute substantially to the mass measurement of planet $\mathrm{d}$.

Information about planet eccentricities comes from stability constraints, the TTVs, and the RVs. The eccentricity of planet $\mathrm{e}$ is not constrained by either the TTVs or the RVs, so its eccentricity varies from 0 to 0.06 (the upper limit from stability requirements). The RVs constrain $e_{b}<0.013$ (95\% confidence, S17). The TTVs constrain $e_{b}<0.02$ (95\% confidence), and the combined RVs + TTVs further constrain $e_{b}<0.011$ (95\% confidence). The RVs alone do not provide a strong constraint for the eccentricity of planet d (S17 fixed $e_{d}=0$ ). The TTVs alone constrain $e_{d}<0.05$ (95\% confidence), and the combined TTVs + RVs constrain $e_{d}<0.025$ (95\% confidence). Thus, the TTVs provide additional information about the small eccentricities of planets $b$ and $d$. The eccentricity of planet $\mathrm{c}$ is determined entirely from RVs because the planet is dynamically decoupled from the inner planetary system.

Thus, combining the TTVs and RVs provides more information about masses and eccentricities than either data set does alone. We discuss the physical importance of having precise measurements for the masses and eccentricities below.

\subsection{Masses and Densities of the Planets}

WASP-47 is unusual in that the masses of its planets span almost two orders of magnitude. The low-mass planets e and d are shown in density-radius and mass-radius plots for small planets (see Figure 8). The lowest-mass planet, WASP-47 e, is $9.1 \pm 1.0 M_{\oplus}$. At $1.8 R_{\oplus}$, this planet has a density of $9.22 \pm 1.06 \mathrm{~g} \mathrm{~cm}^{-3}$. Planets larger than approximately $1.5 R_{\oplus}$ are unlikely to be rocky (Weiss \& Marcy 2014; Rogers 2015). Yet, planet $\mathrm{e}$ is small and dense enough that a rocky composition is likely based on an extrapolation of the empirical relationship for rocky planets smaller than 1.5 Earth radii (Weiss \& Marcy 2014) and theoretical predictions of planet mass and radius for an Earth-like composition (Seager et al. 2007). However, the density of planet e is also consistent with slightly lower densities that might correspond to a rocky interior overlaid with a thin, low-mass envelope of high mean molecular weight materials. Such a composition has also been 


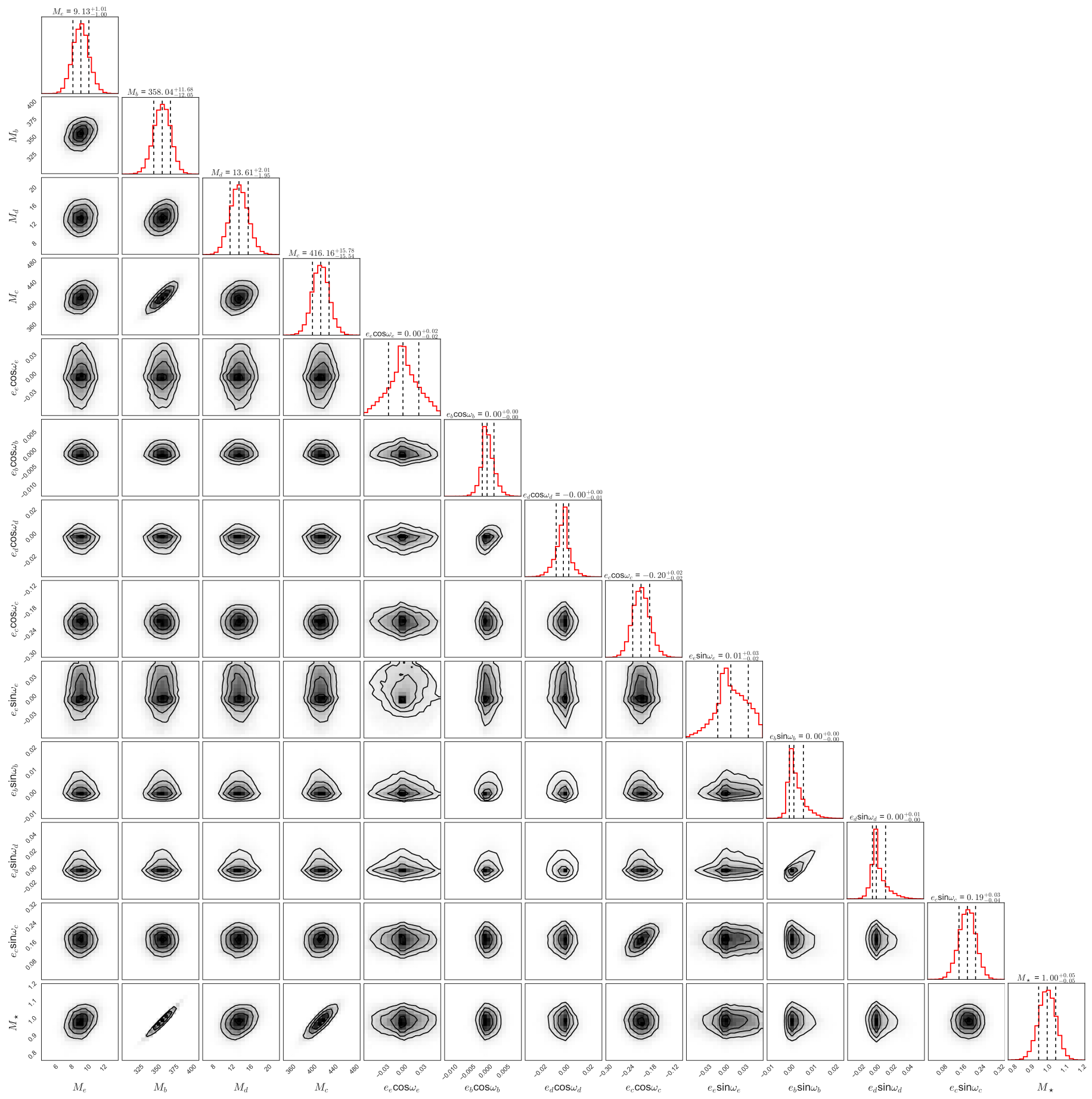

Figure 6. Mass and eccentricity posteriors of the WASP-47 planets based on a simultaneous fit to the TTVs and RVs (see Equation (3)) using the analytic TTV package TTVFaster and the Keplerian RV package radvel. The masses, orbital periods, times of transit, $\sqrt{e} \cos \omega, \sqrt{e} \sin \omega$, and stellar mass were allowed to vary. The MCMC chains shown are available as data behind the figure. The data used to create this figure are available.

hypothesized for 55 Cancri e, which has a very similar mass, radius, and bulk density to WASP-47 e (Lopez 2016, S17). Like 55 Cnc e, WASP-47 e is also an ultra-short period planet cohabiting an orbital system with giant planets, which underscores the question of how planetary system architecture and planet compositions are related.

By contrast, WASP-47 d, which is $3.6 R_{\oplus}$, has a mass of $13.6 \pm 2.0 M_{\oplus}$. This is a slightly higher mass than what was reported in S17 because the TTVs add mass information. The additional information from the TTVs also narrows the mass posterior, shrinking the uncertainty from 2.7 to $2.0 M_{\oplus}$. The density of WASP- $47 \mathrm{~d}$ is $1.63 \pm 0.23 \mathrm{~g} \mathrm{~cm}^{-3}$, making it a highdensity member of the population of sub-Neptune-sized planets with volatile envelopes. The mass-radius diagram in Figure 8 shows that the mass, radius, and density of WASP-47 d make it one of the most Neptune-like planets discovered to date. While its composition could be explained as a two-layer model of a $\mathrm{H} / \mathrm{He}$ envelope atop a silicate-iron core, a Neptune-like 
Table 3

Dynamical Parameters from a Simultaneous Fit to TTVs (ttvfaster) + RVs (Keplerian)

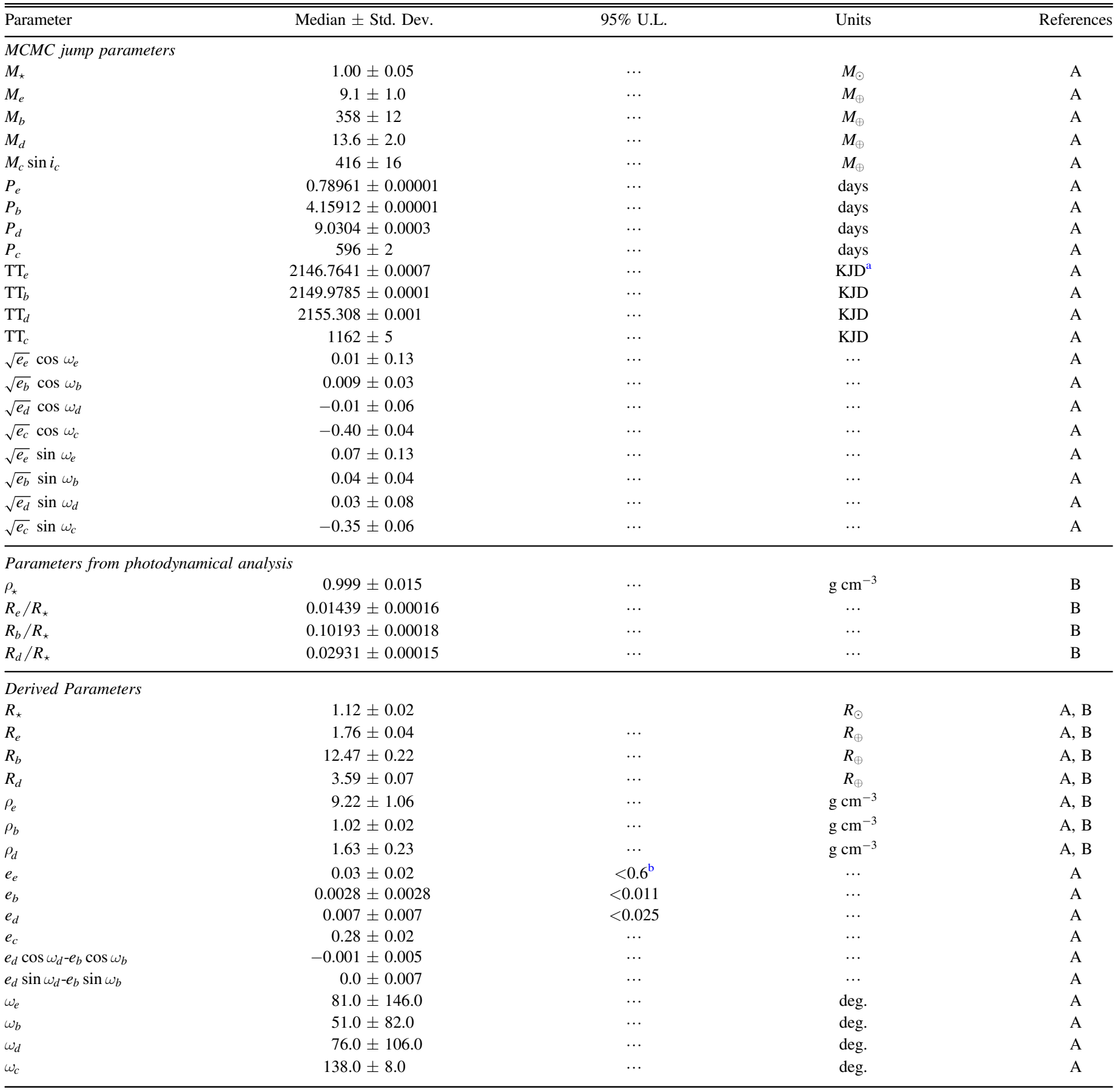

Notes. Results from the MCMC analysis of the TTVs + RVs. The columns are: parameter, median value plus-or-minus standard deviation, 95\% upper limit (if interesting), and units. A-Derived in this analysis. B-incorporating $\rho_{\star}=0.999 \pm 0.015$ from Almenara et al. (2016).

${ }^{\mathrm{a}} \mathrm{KJD}=\mathrm{BJD}-2454833.0$.

${ }^{\mathrm{b}}$ Note that the upper limit on the eccentricity of planet $\mathrm{e}$ is determined from orbital stability requirements, not the measurements.

composition that includes a thick layer of super-ionic water might also explain the bulk properties of WASP-47 $\mathrm{d}$.

WASP-47 $\mathrm{b}$ is a Jupiter-mass planet $\left(358 \pm 12 M_{\oplus}\right)$ that receives $440 \pm 70$ times as much incident stellar irradiation as the Earth does. At $13.11 \pm 0.89 R_{\oplus}$, the planet has a typical density $\left(1.02 \pm 0.02 \mathrm{~g} \mathrm{~cm}^{-3}\right)$ for its mass and incident stellar flux (see Figure 9), consistent with various theories (Fortney \& Nettelmann 2010; Batygin et al. 2011, and references therein) that stellar irradiation inflates the planet and/or prevents the planet from cooling.

\subsection{Eccentricities of the Planets}

The orbits of the three inner planets are profoundly circular. B15 found that eccentricities of $<0.06$ were required for stability. Here, we tighten the eccentricities to $e_{b}<0.011$ and $e_{d}<0.025$ 

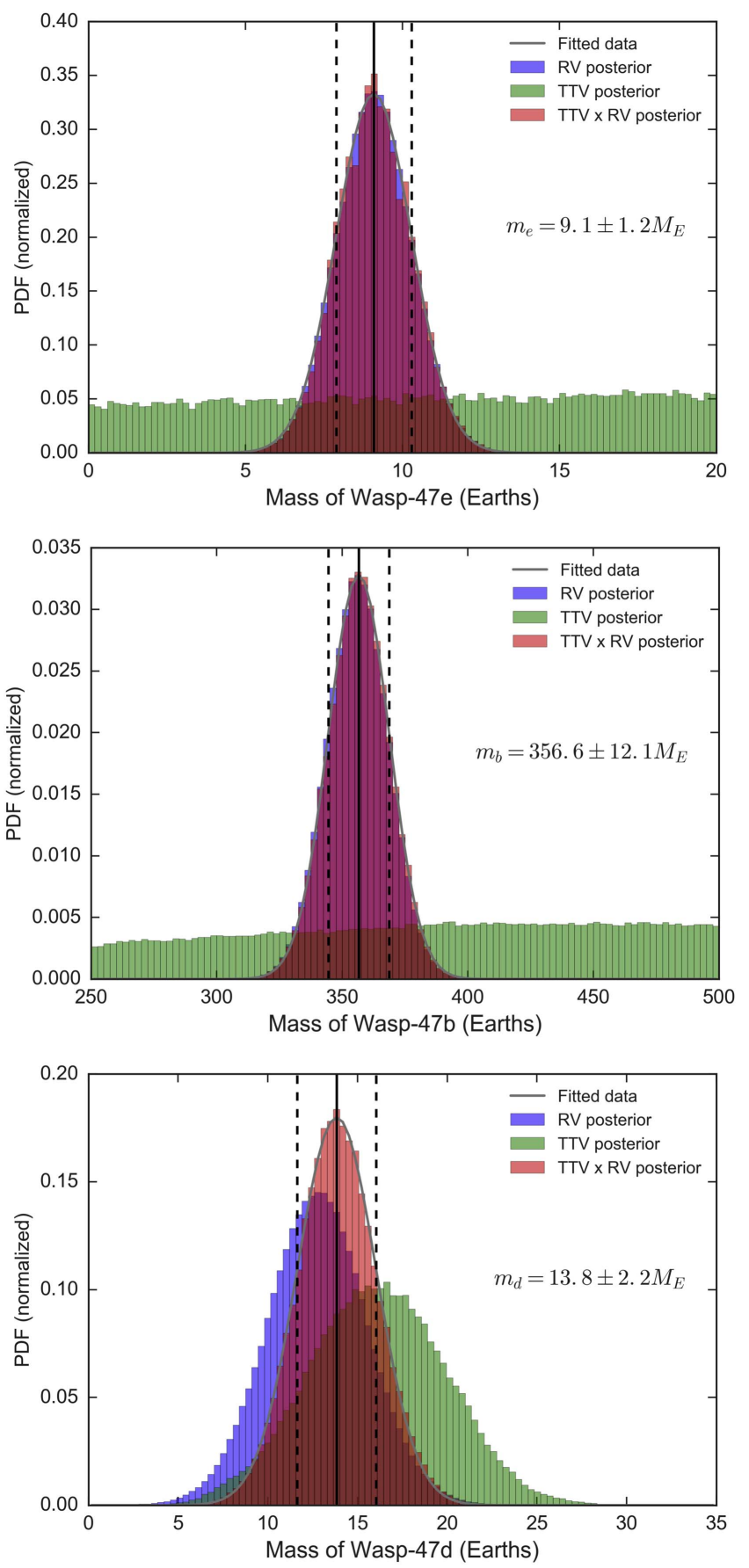

Figure 7. Top: posteriors of the mass of WASP-47 e from analyses of the RVs only (blue), the TTVs only (green, using the $N$-body integrator TTVFast), and their product (i.e., IMP, red). The gray line shows a Gaussian fit to the IMP, with the mean (solid black line) and $1 \sigma$ interval (dashed black lines) shown. Middle: same as the top, but for WASP-47 b. Bottom: same as the top, but for WASP-47 d. Note that the result of computing the IMP for planet d is in good agreement with the simultaneous RV+TTV analysis $\left(13.6 \pm 2.0 M_{\oplus}\right)$.

(95\% confidence). In the highest-eccentricity cases for planets $\mathrm{b}$ and $\mathrm{d}$, they tend to be apsidally aligned. We compute $e_{d} \cos \omega_{d}-e_{b} \cos \omega_{b}=-0.001 \pm 0.005$ and $e_{d} \sin \omega_{d}-$ $e_{b} \sin \omega_{b}=0.0 \pm 0.007$.

Although the tidal circularization timescale for planet e is only $\sim 10^{4}-10^{5}$ years, depending on the tidal $Q$ value for the planet, our $N$-body analysis revealed that the neighboring giant planet (b) perturbs the eccentricity of planet e on a timescale of
1.26 days. This timescale happens to be related to the orbital periods of both e and b by $1 / P_{\text {kick }}=\left(1 / P_{e}+2 / P_{b}\right)^{-1}$. Since there is no commensurability between the orbital periods of $b$ and e, we expect that over long timescales, the kicks from planet $b$ average out, so the argument of periastron of planet $e$ should not have a preferred value. However, planet e could still have an average eccentricity that is higher than zero. Likewise, planet $\mathrm{d}$ should not be assumed to have zero eccentricity because it exhibits TTVs. Because planet $d$ is near the $2: 1$ resonance with planet $b$, its argument of periastron circulates at the frequency of the TTV super-period.

The very low eccentricities of the WASP-47 planets are remarkably like those of the solar system planets (see Figure 10). The average eccentricity of the detected planets in WASP-47 is <0.09; in the solar system, the average eccentricity of the planets and Pluto is 0.08 .

\subsection{Observational Constraints on Formation Theories}

Compact systems like the WASP-47 inner planets might form in situ from protoplanetary disks that are more massive than the minimum mass solar nebula (Chiang \& Laughlin 2013). Scenarios in which either gas-poor sub-Neptunes or gas-rich Jupiters form in situ have been proposed (Lee et al. 2014; Lee \& Chiang 2015; Batygin et al. 2016; Lee \& Chiang 2016). If all the WASP-47 planets formed in situ from the same nebular material, the challenge is to explain how WASP-47 b managed to achieve runaway growth, whereas its immediate neighbors remained gaspoor. Whether a Jupiter-mass or sub-Saturn mass planet forms depends on the core mass, atmospheric opacity, and the disk lifetime (Hori \& Ikoma 2011; Venturini et al. 2015). Cores of mass $\sim 5-15 M_{\oplus}$ and larger undergo runaway gas accretion (with the exact value of critical core mass depending on the atmospheric opacities, metallicities and the disk gas dissipation timescale; Batygin et al. 2016; Lee \& Chiang 2016). However, gas-poor sub-Neptunes are formed instead of giant planets if the cores form and accrete their envelopes in a gas-poor (transitional) disk. The formation of sub-Neptune cores by giant impact requires at least four orders of magnitude of gas-depletion with respect to the minimum mass extrasolar nebula (Lee \& Chiang 2016), leaving $\sim 0.3 M_{\oplus}$ of gas in the disk. This gas budget is insufficient to form the $\sim 300 M_{\oplus}$ of gas in WASP-47 b.

A modification of in situ formation is inside-out growth via pebble accretion, in which pebbles are transported inward through the disk until they are stopped by a pressure maximum at the boundary between the magneto-rotational instability (MRI) zone and the magnetic dead zone (Chatterjee \& Tan 2014). At this boundary, the pebbles may become Toomre unstable or may coalesce via core accretion. As the gas disk clears and the boundary between the MRI and dead zone moves outward, the site of planet formation gradually moves out through the disk. Because the hot Jupiter is situated between two sub-Neptunes, the accretion rate of the disk would likely need to increase by roughly an order of magnitude, and then decrease again, to explain the high mass of the hot Jupiter planet compared to its neighbors. Furthermore, the efficacy of forming hot Jupiters via inside-out planet formation has not been well studied.

Alternatively, the planets could have formed elsewhere in the disk and then migrated via interactions with the disk to their present locations. In both Type I and Type II migration, the gas disk damps planet eccentricity, allowing planets to 
Table 4

Relative Information in Literature Dynamical Analyses

\begin{tabular}{|c|c|c|c|c|c|c|}
\hline Parameter & B15 & A16.1 & A16.2 & S16 & TTVs-Nbody & $\mathrm{RVs}+\mathrm{TTVs}$ \\
\hline$\overline{M_{\star}\left[M_{\odot}\right]}$ & $1.04 \pm 0.08$ & $1.11_{-0.49}^{+0.89}$ & $1.029 \pm 0.031$ & $0.99 \pm 0.05$ & $0.99 \pm 0.05$ & $1.00 \pm 0.05$ \\
\hline$M_{e}\left[M_{\oplus}\right]$ & $<22^{P}$ & $9.1_{-2.9}^{+5.5}$ & $9.1_{-2.9}^{+1.8}$ & $9.11 \pm 1.17$ & $176 \pm 118$ & $9.1 \pm 1.0$ \\
\hline$M_{b}\left[M_{\oplus}\right]$ & $341_{-55}^{+73}$ & $383_{-120}^{+190}$ & $363.8 \pm 8.6$ & $356 \pm 12$ & $549 \pm 252$ & $358 \pm 12$ \\
\hline$M_{d}\left[M_{\oplus}\right]$ & $15.2 \pm 7$ & $16.8_{-7}^{+12}$ & $15.7 \pm 1.1$ & $12.75 \pm 2.70$ & $16.1 \pm 3.8$ & $13.6 \pm 2.0$ \\
\hline$M_{c}\left[M_{\oplus}\right]$ & $\ldots$ & $500_{-190}^{+320}$ & $470_{-100}^{+200}$ & $411 \pm 18$ & $\ldots$ & $416 \pm 16$ \\
\hline$e_{e}$ & $<0.06$ & $<0.11$ & $\cdots$ & $=0^{P}$ & $<0.06^{P}$ & $<0.06^{P}$ \\
\hline$e_{b}$ & $<0.06$ & $<0.01$ & $\cdots$ & $<0.013$ & $<0.05$ & $<0.01$ \\
\hline$e_{d}$ & $<0.06$ & $<0.024$ & $\ldots$ & $=0^{P}$ & $<0.044$ & $<0.025$ \\
\hline$e_{c}$ & $\cdots$ & $0.36 \pm 0.12$ & $\cdots$ & $0.27 \pm 0.04$ & $\cdots$ & $0.28 \pm 0.02$ \\
\hline
\end{tabular}

Note. B15-Becker et al. (2015), K2 TTVs, fixed $P$, TT for each planet, $10 \mathrm{Myr}$ stability-enforced. A16.1-Almenara et al. (2016), photodynamical analysis of $K 2$ TTVs and 71 RVs. A16.2—including stellar models. S16-Sinukoff et al. 2016, 118 RVs. TTVs- $N$ body-TTVFast $N$-body analysis (presented herein), $K 2$ TTVs. RVs+TTVs—simultaneous analysis of K2 TTVs and 118 RVs. All upper limits are at 95\% confidence. $P$-results come from a prior.

maintain circular orbits in a manner consistent with the nearly circular orbits of the three inner planets. However, slow migration can trap the planets in mean motion resonances. While WASP-47 b and $d$ are near the 2:1 mean motion resonance, they are not trapped. Thus, their migration history must either include a mechanism to prevent planets $b$ and $d$ from entering the 2:1 resonance, or remove them from the resonance (e.g., Adams et al. 2008; Goldreich \& Schlichting 2014). In particular, Deck \& Batygin (2015) find that if the inner planet of a pair near a first-order mean motion resonance is more massive (as is the case for WASP-47 b and d), escape from resonance is unlikely. Furthermore, migration in the disk does not explain the eccentricity of planet $\mathrm{c}$ $(0.28 \pm 0.02$. Also, planets e and $\mathrm{d}$ would need to migrate through the disk without accreting gas.

Planet-planet scattering and Kozai-Lidov oscillations are big-body (as opposed to gas-and-dust) migration mechanisms. After the gas disk has dissipated, these mechanisms can introduce moderate to high eccentricities in the orbits, potentially accounting for the moderate eccentricity of planet c. Kozai-Lidov oscillations are initiated only when two planets have mutual inclinations of at least $40^{\circ}$. The planets swap angular momentum, causing dramatic variations in the inclinations and eccentricities of the planets over time (Kozai 1962; Lidov 1962). The strongest lines of evidence for past Kozai-Lidov interactions would be (1) an observed large mutual inclination between planet $\mathrm{c}$ and the inner solar system, and/or (2) a non-zero obliquity between planet b and the stellar spin axis. Sanchis-Ojeda et al. (2015) ruled out a significant spin-orbit misalignment for planet $b$, but the degeneracy between stellar $v \sin i$ and the planet-star obliquity allows moderate misalignments $\left(|\lambda|<48^{\circ}\right.$ with $1 \sigma$ confidence). If planet $\mathrm{c}$ is coplanar and the spin-orbit alignments of the planets are small, a coplanar, high-eccentricity migration (HEM) scenario such as the one proposed in Petrovich (2015) might best explain the present locations of the planets. However, the current nearly circular, coplanar orbits of the inner planetary system are not consistent with a past modest eccentricity and/or inclination for planet b. Recall from the stability analysis in B15 that the nearly all of the scenarios in which the eccentricity of planet $b$ exceeds 0.06 become unstable within $10 \mathrm{Myr}$. Therefore, although high-eccentricity migration mechanisms can explain the present positions of planets $b$ and $c$, such mechanisms would have destroyed the small, close-in planets. Indeed, simulations of giant planets migrating inward find that the giant planets collide with lowmass inner planets in both high-eccentricity (Mustill et al. 2015) and low-eccentricity (Batygin \& Laughlin 2015) scenarios.

\subsection{The Two-stage Planet Formation Hypothesis}

One way to explain the present orbits and compositions of the WASP-47 planets is that the planets did not all form at the same time. As a point of reference, consider the solar system. In our own solar system, the giant planets must have formed early (within 1 to $10 \mathrm{Myr}$ ), when the protoplanetary disk was still gas-rich (de Pater \& Lissauer 2001). In contrast, the formation of terrestrial-mass planets by planetesimal accretion can take as long as $\sim 10^{8}$ years in $N$-body simulations and is more efficient in gas-poor disks where the planetesimal eccentricities can grow, leading to more collisions (Lissauer 1987; Pollack et al. 1996; Ida \& Lin 2004; Lee \& Chiang 2016). Thus, it is possible that the solar system giant planets formed and migrated to their present locations before the terrestrial planets formed. The Nice Model demonstrates that early formation and migration of Jupiter and Saturn can reproduce the current orbital architectures of the gas giants and ice giants while also accounting for the period of Late Heavy Bombardment on the terrestrial planets, and Trojan asteroids (Gomes et al. 2005; Morbidelli et al. 2005; Tsiganis et al. 2005). The Grand Tack Model shows that a reversal in the direction of Jupiter's migration due to torques from Saturn can explain the small size of Mars and detailed compositional features of the asteroids (Walsh et al. 2011; Morbidelli et al. 2012). Both of these models feature two stages of planet formation:

1. Early giant planet growth, combined with disk and/or planet-induced migration, produces the current compositions of giant planets and sculpts the radial distribution of planetesimals.

2. When or after the gas disk clears, planetesimal accretion proceeds in the sculpted planetesimal disk, resulting in the formation of low-mass, predominantly rocky planets.

Likewise, the formation and evolution of the planets in WASP-47 might be best explained by a two-stage process. The giant planets in the WASP-47 system might have formed early in the gas-rich disk and exchanged energy with each other, additional bodies, or the disk to migrate to their present locations. If the gas disk cleared while WASP-47 b and c 

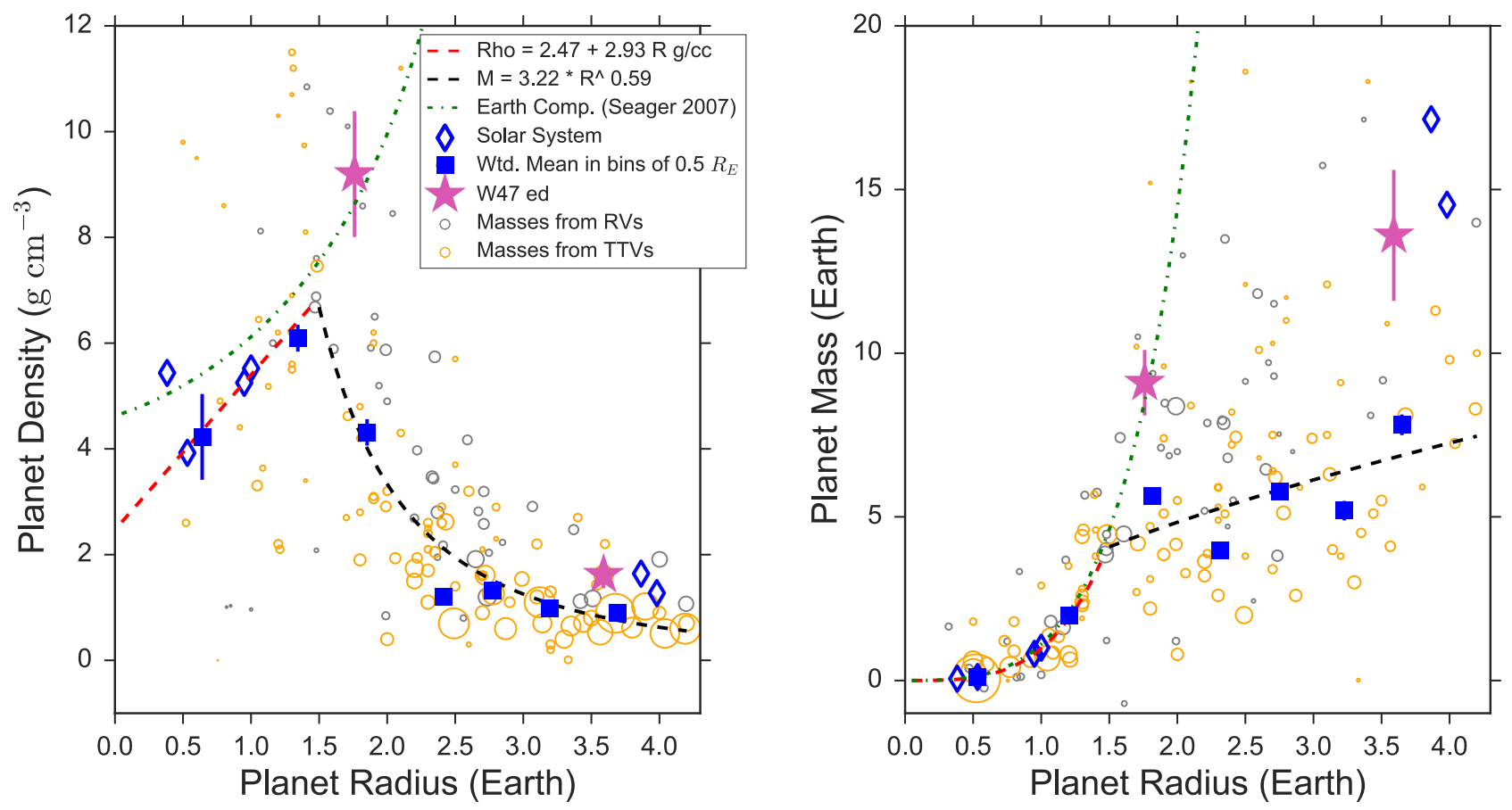

Figure 8. Left: planet density vs. planet physical radius for 94 transiting planets smaller than $4.2 R_{\oplus}$. The gray circles have masses determined from RVs; the gold circles have masses determined from TTVs. The size of the circle corresponds to $1 / \sigma_{\rho}^{2}$. Blue squares show the weighted mean density in bins of $0.5 R_{\oplus}$ to guide the eye. The blue diamonds are the solar system planets. The red dashed line is an empirical linear fit to planet density vs. radius for the exoplanets and solar system planets smaller than $1.5 R_{\oplus}$, extended to predict the densities of potentially rocky planets larger than $1.5 R_{\oplus}$. For comparison, we show the predicted density-radius curve for a polytropic equation of state of an Earth-composition planet (Seager et al. 2007, green dotted line). The black line is an empirical power-law fit to planet mass vs. radius for planets larger than $1.5 R_{\oplus}$. WASP-47 e $\left(1.9 R_{\oplus}\right)$ sits on the red line and therefore is consistent with a rocky composition, but could also have a thin volatile envelope. WASP-47 d (3.7 $\left.R_{\oplus}\right)$ has a density that requires significant volatiles, including the possibility of high-density volatiles based on the similarity of its bulk properties to Uranus and Neptune. Right: Same as the left panel, but showing planet mass vs. radius, and the circle sizes correspond to $1 / \sigma_{m}^{2}$.

were finishing their migration, the final years of the giant planets' migration could have influenced the solid material in the disk, exciting protoplanetary solids to higher eccentricities and inducing a second stage of core accretion. Such an epoch could have formed WASP-47 e and d, and perhaps additional yet-undetected low-mass planets dominated by rocky interiors.

\subsection{Predictions of Two-stage Planet Formation}

In WASP-47, the orbital architecture and compositions of the low-mass planets provide insight into the formation history of the giant planets. Because the low-mass planets need a small amount of gas to form (about $0.3 M_{\oplus}$ ), their formation must occur before the gas disk completely dissipates. This puts a deadline on when planet $b$ can finish its migration; it must park at its current location before planets e and $\mathrm{d}$ form (otherwise it destroys them). In other words, planet $\mathrm{b}$ must arrive at its present location before the gas disk dissipates.

Because a gas disk damps planet eccentricities on the timescale of $10^{3}$ years (Papaloizou \& Larwood 2000), high-eccentricity migration cannot proceed in a gas disk. Furthermore, the timescale for type I migration in a gas disk is much faster than the timescale for HEM, so gas disk migration dominates while the gas disk is present. If we consider a mostly gas-depleted disk, we can try to migrate WASP-47 b inward via HEM in a short window of time before the disk completely dissipates. However, some force must circularize planet b's orbit quickly enough to allow the neighboring small planets to form. In the absence of gas, the only circularizing force is tides raised on the planet by the star. The timescale for eccentricity damping due to tides
(Murray \& Dermott 2000, Equation (4.198)) is

$$
\tau_{e}=-\frac{e}{\dot{e}}=\frac{4}{63} \frac{M_{\mathrm{p}}}{M_{\star}}\left(\frac{a}{R_{\mathrm{p}}}\right)^{5} \frac{\mu_{\mathrm{pl}} Q_{\mathrm{pl}}}{2 \pi / P}
$$

where $\mu_{\mathrm{pl}}$ is the ratio of the elastic to gravitational forces in the planet $\approx\left(10^{4} \mathrm{~km} / R_{\mathrm{p}}\right)^{2}$, and $Q_{\mathrm{pl}}$ is the tidal dissipation factor of the planet. For $a=0.05$ au and $Q_{\mathrm{pl}}=10^{6.5}$ (typical for hot Jupiters, Jackson et al. 2008), the circularization time is $\tau_{e} \approx 10^{8}$ years, which is an order of magnitude longer than the disk lifetime. There is not enough time to move WASP-47 b to its present position via HEM and then circularize its orbit before the little planets form. Therefore, a history of HEM is highly unlikely for planet $b$.

On the other hand, planet $b$ could also have formed in situ or nearly in situ in the gas-rich disk. Antonini et al. (2016) find through $N$-body simulations that, for the majority of observed Jupiter pairs (i.e., systems with one Jupiter-mass planet inside 1 $\mathrm{au}$ and one Jupiter-mass planet outside $1 \mathrm{au}$ ), the inner planet is unlikely to have formed beyond 1 au. Thus, it seems probable that WASP-47 $\mathrm{b}$ formed inside $1 \mathrm{au}$, and thus inside the snowline.

Since planet $\mathrm{c}$ has modest eccentricity $(0.28 \pm 0.02$, it almost certainly had some sort of planet-planet interaction in the past. Although it is possible for instabilities in the gas disk to excite giant planet eccentricities, this effect only works for $e<0.1$ and therefore cannot explain the eccentricity of planet $\mathrm{c}$ (Duffell \& Chiang 2015). As described above, planet b is not a likely candidate for pumping the eccentricity of planet c. Thus, additional massive bodies that exchanged angular momentum 

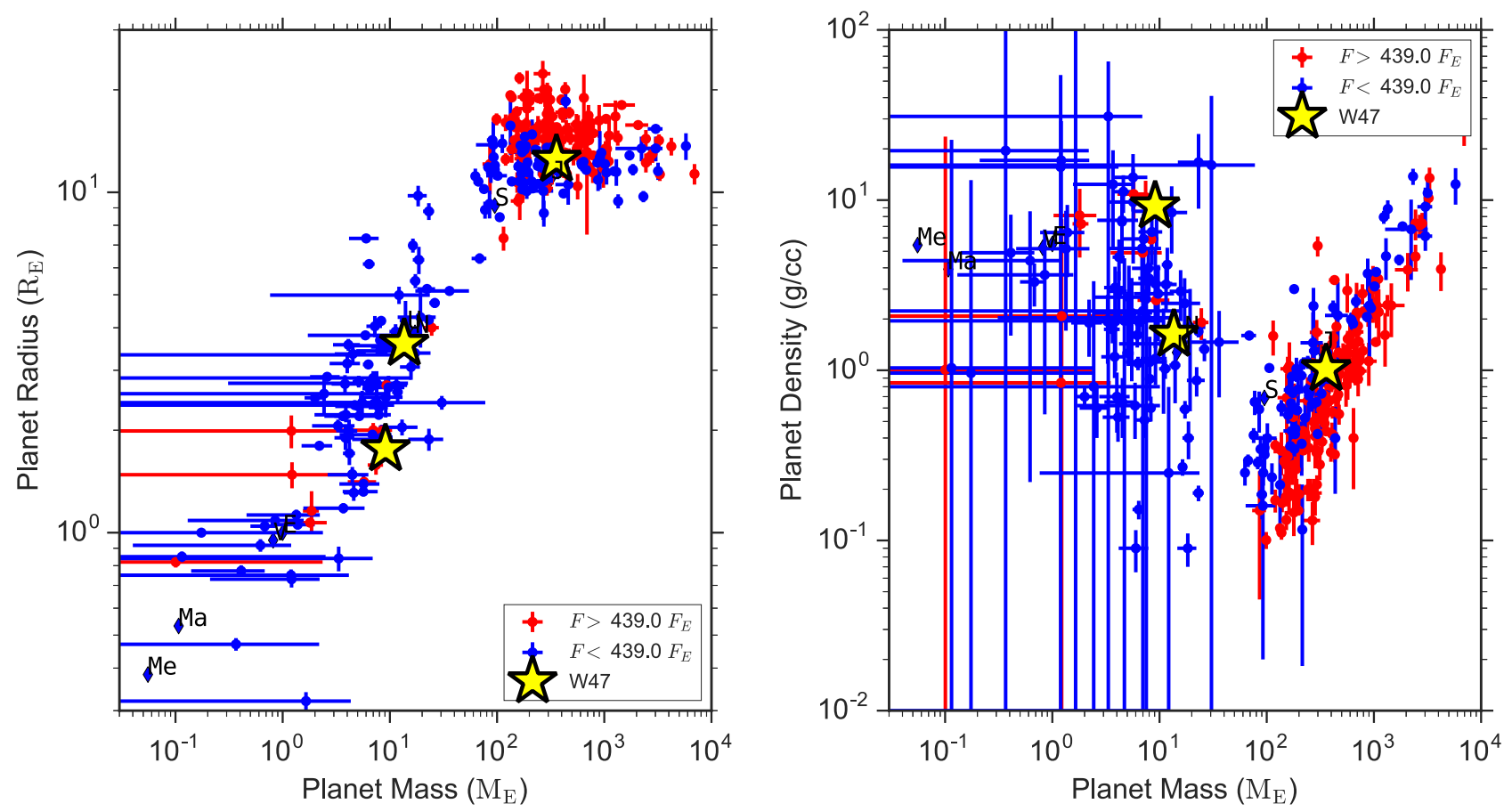

Figure 9. Left: planet radius vs. mass for exoplanets with measured masses and radii, as determined by querying exoplanets.org on 2017 February 19 and including radii and masses from Hadden \& Lithwick (2016) and Gillon et al. (2017). The sample is divided into those that receive more than the median incident flux $\left(F>482 F_{\oplus}\right.$, red points) and those that receive less than the median incident flux $\left(F<482 F_{\oplus}\right.$, blue points). The solar system planets are labeled. The WASP-47 planets are shown (yellow stars, the mass, and radius error bars are smaller than the symbols). The sub-Neptunes WASP-47 e and d are high-density for their size. WASP-47 b is a typical-sized hot Jupiter for its mass and incident flux.

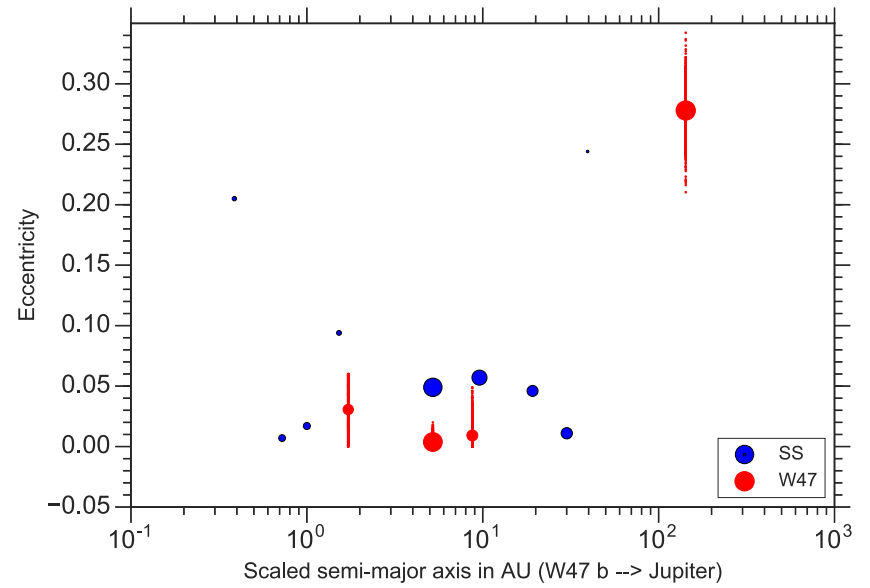

Figure 10. The mean eccentricities of the WASP-47 and solar system planets vs. the orbital distances, scaled such that $a_{b}=a_{J}$ to facilitate comparison. The point size corresponds to $M_{\mathrm{p}}^{1 / 3}$, to illustrate the mass range while keeping all the planets visible. The spread of each WASP-47 planet eccentricity is illustrated with a cloud of 1000 small red points drawn from the eccentricity posterior. The average eccentricity of the W47 planets is comparable to the average eccentricity of the solar system planets, if we include Pluto.

with planet $\mathrm{c}$ in the past might still be present in the WASP-47 system. Alternatively, planet c might have ejected whatever companion pumped its eccentricity.

Based on the above arguments, evidence of two-stage planet formation in WASP-47 could include:

1. a C/O ratio for WASP-47 b, e, and d consistent with formation inside the snowline;

2. alignment of the stellar spin axis with the orbits of the transiting planets, consistent with a coplanar, mostly circular orbital history for planet b;
3. an additional massive planet/sub-stellar/stellar companion with an eccentric orbit that exchanged angular momentum with planet $\mathrm{c}$ in the past.

A two-stage formation mechanism might also best explain the current architectures of other multi-planet systems with diverse planet compositions, such as 55 Cancri and Kepler-89 (KOI-94). An improved census of giant planets accompanying the low-mass planetetary systems discovered by Kepler will enable studies of the occurrence of planetary systems with diverse compositions like that of our solar system, illuminating the dominant physical processes in their formation.

Continued observations of this system and other systems with diverse planet compositions from the James Webb Space Telescope, the WFIRST mission, and other facilities will provide evidence for or against a two-stage planet formation scenario.

\section{Conclusion}

We combine $118 \mathrm{RVs}$ and $108 \mathrm{~K} 2 \mathrm{TTVs}$ to present some of the most precise masses, densities, and orbital dynamics of the WASP-47 planetary system to date. For the transiting inner planetary system, we obtain $M_{e}=9.1 \pm 1.0 M_{\oplus}\left(\rho_{e}=9.22 \pm\right.$ $\left.1.06 \mathrm{~g} \mathrm{~cm}^{-3}\right), M_{b}=358 \pm 12 M_{\oplus}\left(\rho_{b}=1.02 \pm 0.02 \mathrm{~g} \mathrm{~cm}^{-3}\right)$, and $M_{d}=13.6 \pm 2.0 M_{\oplus}\left(\rho_{d}=1.63 \pm 0.23 \mathrm{~g} \mathrm{~cm}^{-3}\right)$.

We place tight upper limits on the eccentricities of the three inner planets: $e_{e}<0.06, e_{b}<0.011$, and $e_{d}<0.025(95 \%$ confidence). The mean eccentricity of the WASP-47 planets $(0.09)$ is very similar to the mean eccentricity of the solar system planets plus Pluto (0.08).

Of the four planets, only WASP-47 e has bulk properties consistent with a rocky composition. WASP-47 $\mathrm{d}$ is a subNeptune-sized planet that is just a little smaller than Neptune but has the density of Neptune, meaning that its bulk properties 
are consistent with a Neptune-like composition. However, WASP-47 d could also be a rocky interior overlaid with a hydrogen-rich, water-poor envelope. WASP-47 b is a hot Jupiter with a typical size for its mass and incident stellar flux.

The non-resonant architecture, diverse masses, and profoundly circular orbits in the WASP-47 system resemble our own solar system. We briefly review the highlights of in situ planet formation, inside-out planet formation, disk migration, and high-eccentricity migration, noting that none of these mechanisms alone can reproduce all the physical attributes of the WASP-47 system. We propose that WASP-47, like the solar system, formed in two stages. In stage one, the giant planets formed in a gas-rich disk and migrated via disk migration to their present locations. In stage two, the highdensity sub-Neptunes formed in situ in a gas-poor environment.

L.M.W. acknowledges the Trottier Family Foundation for their support. K.D. acknowledges the support of the JCPA fellowship at Caltech. E.S. is supported by a post-graduate scholarship from the Natural Sciences and Engineering Research Council of Canada. E.A. acknowledges support from NASA grants NNX13AF20G, NNX13AF62G, and the NASA Astrobiology Institutes Virtual Planetary Laboratory, supported by NASA under cooperative agreement NNH05ZDA001C. A.W.H. acknowledges support from a NASA Astrophysics Data Analysis Program grant, support from the $K 2$ Guest Observer Program, and a NASA Key Strategic Mission Support Project. We thank Simon Walker for his help and generosity in using ttvfast-python. The authors thank Geoff Marcy, Eva Culakova, Daniel Fabrycky, Jack Lissauer, Jason Rowe, the Kepler-TTV working group, and the KITP Planet Formation and Dynamics workshop for useful discussions. We thank NASA and the Kepler team for the outstanding photometry that contributed to this paper. The authors wish to extend special thanks to those of Hawaiian ancestry on whose sacred mountain of Maunakea we are privileged to be guests. Without their generous hospitality, the Keck observations analyzed herein would not have been possible.

Facilities: Keck:I (HIRES), Kepler.

Software: TTVFaster (Agol \& Deck 2016a, 2016b), TTVFast (Deck et al. 2014), ttvfast-python (https://github. com/mindriot101/ttvfast-python), emcee (Foreman-Mackey et al. 2013), lmfit (Newville et al. 2014), radvel (https:// github.com/California-Planet-Search/radvel).

\section{References}

Adams, F. C., Laughlin, G., \& Bloch, A. M. 2008, ApJ, 683, 1117 Agol, E., \& Deck, K. 2016a, ApJ, 818, 177

Agol, E., \& Deck, K. 2016b, TTVFaster: First Order Eccentricity Transit Timing Variations (TTVs), Astrophysics Source Code Library, ascl:1604.012

Almenara, J. M., Díaz, R. F., Bonfils, X., \& Udry, S. 2016, A\&A, 595, L5 Antonini, F., Hamers, A. S., \& Lithwick, Y. 2016, AJ, 152, 174

Batalha, N. M., Rowe, J. F., Bryson, S. T., et al. 2013, ApJS, 204, 24

Batygin, K., Bodenheimer, P. H., \& Laughlin, G. P. 2016, ApJ, 829, 114

Batygin, K., \& Laughlin, G. 2015, PNAS, 112, 4214

Batygin, K., Stevenson, D. J., \& Bodenheimer, P. H. 2011, ApJ, 738, 1

Becker, J. C., Vanderburg, A., Adams, F. C., Rappaport, S. A., \& Schwengeler, H. M. 2015, ApJL, 812, L18

Borucki, W. J., Koch, D. G., Brown, T. M., et al. 2010, ApJL, 713, L126

Brooks, S. P., \& Gelman, A. 1998, Journal of Computational and Graphical Statistics, 7, 434

Bryan, M. L., Knutson, H. A., Howard, A. W., et al. 2016, ApJ, 821, 89

Burke, C. J., Christiansen, J. L., Mullally, F., et al. 2015, ApJ, 809, 8

Chambers, J. E. 1999, MNRAS, 304, 793

Chatterjee, S., \& Tan, J. C. 2014, ApJ, 780, 53
Chiang, E., \& Laughlin, G. 2013, MNRAS, 431, 3444

Dai, F., Winn, J. N., Arriagada, P., et al. 2015, ApJL, 813, L9

de Pater, I., \& Lissauer, J. J. 2001, Planetary Sciences (2nd ed.; Cambridge: Cambridge Univ. Press)

Deck, K. M., Agol, E., Holman, M. J., \& Nesvorný, D. 2014, ApJ, 787, 132

Deck, K. M., \& Batygin, K. 2015, ApJ, 810, 119

Dotter, A., Chaboyer, B., Jevremović, D., et al. 2008, ApJS, 178, 89

Dressing, C. D., \& Charbonneau, D. 2015, ApJ, 807, 45

Duffell, P. C., \& Chiang, E. 2015, ApJ, 812, 94

Fabrycky, D. C., Lissauer, J. J., Ragozzine, D., et al. 2014, ApJ, 790, 146

Fang, J., \& Margot, J.-L. 2012, ApJ, 761, 92

Foreman-Mackey, D., Hogg, D. W., Lang, D., \& Goodman, J. 2013, PASP, 125,306

Fortney, J. J., \& Nettelmann, N. 2010, SSRv, 152, 423

Fressin, F., Torres, G., Charbonneau, D., et al. 2013, ApJ, 766, 81

Gelman, A., \& Rubin, D. B. 1992, StaSc, 7, 457

Gillon, M., Triaud, A. H. M. J., Demory, B.-O., et al. 2017, Natur, 542, 456

Gladman, B. 1993, Icar, 106, 247

Goldreich, P., \& Schlichting, H. E. 2014, AJ, 147, 32

Gomes, R., Levison, H. F., Tsiganis, K., \& Morbidelli, A. 2005, Natur, 435,466

Hadden, S., \& Lithwick, Y. 2016, arXiv:1611.03516

Hellier, C., Anderson, D. R., Collier Cameron, A., et al. 2012, MNRAS, 426, 739

Hori, Y., \& Ikoma, M. 2011, MNRAS, 416, 1419

Howard, A. W., Marcy, G. W., Bryson, S. T., et al. 2012, ApJS, 201, 15

Ida, S., \& Lin, D. N. C. 2004, ApJ, 604, 388

Jackson, B., Greenberg, R., \& Barnes, R. 2008, ApJ, 678, 1396

Kipping, D. M. 2014, MNRAS, 440, 2164

Koch, D. G., Borucki, W. J., Rowe, J. F., et al. 2010, ApJL, 713, L131

Kozai, Y. 1962, AJ, 67, 591

Lee, E. J., \& Chiang, E. 2015, ApJ, 811, 41

Lee, E. J., \& Chiang, E. 2016, ApJ, 817, 90

Lee, E. J., Chiang, E., \& Ormel, C. W. 2014, ApJ, 797, 95

Lidov, M. L. 1962, P\&SS, 9, 719

Lissauer, J. J. 1987, Icar, 69, 249

Lissauer, J. J., Marcy, G. W., Bryson, S. T., et al. 2014, ApJ, 784, 44

Lissauer, J. J., Marcy, G. W., Rowe, J. F., et al. 2012, ApJ, 750, 112

Lithwick, Y., Xie, J., \& Wu, Y. 2012, ApJ, 761, 122

Lopez, E. D. 2016, arXiv:1610.01170

Lovis, C., \& Fischer, D. 2010, in Exoplanets, Radial Velocity Techniques for Exoplanets, ed. S. Seager (Tucson, AZ: Univ. Arizona Press), 27

Morbidelli, A., Levison, H. F., Tsiganis, K., \& Gomes, R. 2005, Natur, 435,462

Morbidelli, A., Lunine, J. I., O’Brien, D. P., Raymond, S. N., \& Walsh, K. J. 2012, AREPS, 40, 251

Murray, C. D., \& Correia, A. C. M. 2010, in Exoplanets, Keplerian Orbits and Dynamics of Exoplanets, ed. S. Seager (Tucson, AZ: Univ. Arizona Press), 15

Murray, C. D., \& Dermott, S. F. 2000, Solar System Dynamics (Cambridge: Cambridge Univ. Press), 321

Mustill, A. J., Davies, M. B., \& Johansen, A. 2015, ApJ, 808, 14

Neveu-VanMalle, M., Queloz, D., Anderson, D. R., et al. 2016, A\&A, 586, A93

Newville, M., Stensitzki, T., Allen, D. B., \& Ingargiola, A. 2014, LMFIT: NonLinear Least-Square Minimization and Curve-Fitting for Python, doi: 10 5281 /zenodo. 11813

Papaloizou, J. C. B., \& Larwood, J. D. 2000, MNRAS, 315, 823

Petigura, E. A., Howard, A. W., \& Marcy, G. W. 2013a, PNAS, 110, 19273

Petigura, E. A., Marcy, G. W., \& Howard, A. W. 2013b, ApJ, 770, 69

Petrovich, C. 2015, ApJ, 805, 75

Pollack, J. B., Hubickyj, O., Bodenheimer, P., et al. 1996, Icar, 124, 62

Rogers, L. A. 2015, ApJ, 801, 41

Rowe, J. F., Bryson, S. T., Marcy, G. W., et al. 2014, ApJ, 784, 45

Sanchis-Ojeda, R., Rappaport, S., Winn, J. N., et al. 2013, ApJ, 774, 54

Sanchis-Ojeda, R., Winn, J. N., Dai, F., et al. 2015, ApJL, 812, L11

Seager, S., Kuchner, M., Hier-Majumder, C. A., \& Militzer, B. 2007, ApJ, 669, 1279

Sinukoff, E., Howard, A. W., Petigura, E. A., et al. 2017, AJ, 153, 70

Steffen, J. H., Ragozzine, D., Fabrycky, D. C., et al. 2012, PNAS, 109, 7982

Tsiganis, K., Gomes, R., Morbidelli, A., \& Levison, H. F. 2005, Natur, 435,459

Venturini, J., Alibert, Y., Benz, W., \& Ikoma, M. 2015, A\&A, 576, A114

Walsh, K. J., Morbidelli, A., Raymond, S. N., O’Brien, D. P., \& Mandell, A. M. 2011, Natur, 475, 206

Weiss, L. M., \& Marcy, G. W. 2014, ApJL, 783, L6 